\title{
Multi-stage consistency optimization algorithm for decision making with incomplete probabilistic linguistic preference relation
}

\author{
Peng Wang ${ }^{\mathrm{a}, \mathrm{b}}$, Peide $\mathrm{Liu}^{\mathrm{a}}{ }^{*}$, Francisco Chiclana ${ }^{\mathrm{b}, \mathrm{c}}$ \\ aschool of Management Science and Engineering, Shandong University of Finance and Economics, Shandong, \\ China \\ bInstitute of Artificial Intelligence, De Montfort University, Leicester, UK \\ cAndalusian Research Institute on Data Science and Computational Intelligence, University of Granada, Granada, \\ Spain \\ *Corresponding author: Tel: +86-531-82222188, E-mail: Peide.liu@gmail.com
}

\begin{abstract}
Incomplete probabilistic linguistic term sets (InPLTSs) can effectively describe the qualitative pairwise judgment information in uncertain decision-making problems, making them suitable for solving real decision-making problems under time pressure and lack of knowledge. Thus, in this study, an optimization algorithm is developed for preference decision-making with the incomplete probabilistic linguistic preference relation (InPLPR). First, to fully investigate the ability of InPLTSs to express uncertain information, they are divided into two categories. Then, a two-stage mathematical optimization model based on an expected multiplicative consistency for estimating missing information is constructed, which can obtain the complete information more scientifically and effectively than some exiting methods. Subsequently, for the InPLPR with unacceptable consistency, a multi-stage consistency-improving optimization model is proposed for improving the consistency of the InPLPR by minimizing the information distortion and the number of adjusted linguistic terms, which can also minimize the uncertainty of the relationship as small as possible. Afterward, to rank all the alternatives, a mathematical model for deriving the priority weights of the alternatives is constructed and solved, which can obtain the priority weight conveniently and quickly. A decision-making algorithm based on the consistency of the InPLPR is developed, which involves estimating missing information, checking and improving the consistency, and ranking the alternatives. Finally, a numerical case involving the selection of excellent students is presented to demonstrate the application of the proposed algorithm, and a detailed validation test and comparative analysis are presented to highlight the advantages of the proposed algorithm.
\end{abstract}

Keywords: preference decision-making; incomplete probabilistic linguistic preference relation; expected multiplicative consistency; mathematical optimization model; excellent student selection.

\section{Introduction}

Generally, there are many ways to identify the best selection among multiple alternatives, such as multi-attribute decision-making (MADM) [18, 23], which can obtain a comprehensive evaluation result for each alternative based on the evaluation values of multiple attributes. However, to better conform to humans' evaluation habits and minimize the decision-making time and cost, the preference relation, which is also known as the analytic hierarchy process (AHP), was proposed by Saaty [25]. For pairwise comparison between alternatives, preference decision-making (PDM) allows experts to generate reasonable preference information about each object based on specific criteria. The process of pairwise comparison is similar to the practical decision-making process and the mental judgement process of humans. Thus, it has become a popular evaluation method for presenting judgment information and has attracted extensive research attention $[6,27,29,38,45,47]$.

However, considering the uncertainty of the evaluation object and the ambiguity of human subjective 
judgment, it is inadequate for experts to describe preference information using crisp numbers in reality. relation with a fuzzy set. Subsequently, various forms of fuzzy preference relations have been investigated, such as intuitionistic fuzzy preference relations [36], hesitant fuzzy preference relations [42], and probabilistic hesitant fuzzy preference relations [50]. Herrera and Herrera-Viedma [12] reported that when experts are unable to provide quantitative fuzzy preference information with exact numbers, they can express qualitative preferences using the linguistic variables (LVs) proposed by Zadeh [44]. With the continuous development of linguistic representation models, novel linguistic forms are emerging, which compensate for the shortcoming that traditional linguistic term sets (LTSs) cannot express complex decision-making information effectively. Hence, linguistic preference relations are also widely studied, and numerous research results have been reported, e.g., 2-tuple linguistic preference relations [6], hesitant fuzzy linguistic preference relations (HFLPRs) [14], and distribution linguistic preference relations [45].

Among the linguistic preference relations, the most prominent is the probabilistic linguistic preference relation (PLPR) introduced by Zhang et al. [48]. This is because the probabilistic LTSs (PLTSs) introduced in [23] have the advantage of expressing complex linguistic evaluation information, that is, they can not only present some hesitation information with several linguistic terms (LTs) but also reflect the importance (or certainty) of each possible LT in the form of a probability [18, 37]. Moreover, PLTSs are advantageous compared with linguistic distribution assessments (LDAs) [45], the possibility distribution for HFLTS (PD-HFLTS) [39], and proportional hesitant fuzzy LTSs (PHLTSs) [2] when dealing with complex situations involving partial ignorance, because they allow the sum of probabilities of all possible LTs to be $<1$. Incomplete probabilistic linguistic information is particularly common in complex decision-making environments, e.g., the absence of expert opinions within a group. Thus, PLTSs exhibit considerable flexibility and comprehensiveness in expressing hesitant and ignorant qualitative linguistic evaluations.

In view of the outstanding advantages of PLTSs for expressing uncertain information, a large amount of research has recently been performed on PLPRs. The research on probabilistic linguistic preference decision-making (PLPDM) focuses on the following three aspects.

(1) Research on estimating missing information. The existence of missing information is not rare in practical PLPDM problems owing to experts' insufficient knowledge or the complex decision environment. Therefore, handling the missing information of PLTSs is an important task. In the current PLPR research [46-48], the normalization method for PLTSs proposed by Pang et al. [23] is the most commonly used technique in cases of ignorant information. Additionally, Gao et al. [8, 9] calculated and modified the probability using computer-aided tools in practical application problems with incomplete PLPRs (InPLPRs), e.g., case-based reasoning [9] and emergency fault tree analysis (EFTA) [8]. Song [26] and Song and $\mathrm{Hu}$ [27] established probability computation models for InPLPRs based on the consistency. Additionally, scholars have examined the missing elements in the PLPR matrices via techniques such as additive consistency, social strategies, and Monte Carlo simulation [16, 41].

(2) Research on consistency checking and improvement. Consistency refers to whether the preference information is reasonable and whether there is a logical contradiction. According to the preference relation graph, Zhang et al. [46, 48] introduced additive consistency for PLPRs, and Zhang et al. [47] introduced ordered consistency for PLPRs. Furthermore, they presented automatic iterative algorithms to adjust the consistency. Luo et al. [19] employed the cosine similarity with PLPRs to measure the consistency index; if the PLPR is inconsistent, it is fed back to the experts for correction. Xie et al. [41] 
and $\mathrm{Li}$ et al. [13] simultaneously considered additive consistency and multiplicative consistency for hybrid PLPRs under the definition of transformation functions and proposed some nonlinear optimization models to modify the inconsistent PLPRs. Nie and Wang [21] introduced prospect theory-based consistency recovery strategies to improve inconsistency. Additionally, the checking and improvement of consistency based on the expected values of PLTSs have begun to be widely studied [8, 9, 27].

(3) Research on ranking methods. A scientific and appropriate ranking method is essential for identifying the optimal alternative in PLPDM problems. Integration methods based on the various aggregation operators of PLTSs are the most commonly used to obtain the collective preference value of each alternative and then implement the selection process [15, 16, 21, 46-48]. Additionally, to avoid over-distortion of information in the integration process, scholars have adopted the priority weight to rank the alternatives and output the optimal solution [8, 9, 19, 27, 41].

\section{A. Motivation and contributions}

In the foregoing studies, PLPDM methods were investigated from different perspectives, and useful information regarding PLPRs was obtained. However, there are limitations and shortcomings in the following aspects.

(1) The estimation mechanism for missing information of PLTSs in PLPRs is unreasonable. There are two types of incomplete information in PLTSs: 1) the traditional PLTSs, in which the sum of the probabilities is $<1$, and 2) the occurrence probabilities of multiple or all possible LTs are unknown. For the former, the normalization methods in [46-48] only assign the missing probability to each possible LT according to the existing proportion, which is simple but unscientific. Although the methods in $[26,27]$ can estimate partial and complete missing probabilities of PLTSs using an optimization model, they consider that the missing probability should belong to the existing LTs. In fact, above these approaches is not scientific enough. Because the missing probability is due to the lack of knowledge of an individual decision-maker or the absence of experts' opinions in the group, it does not necessarily belong to the existing LTs. Additionally, for the latter, the methods in $[8,9]$ can calculate the missing probability of LTs through EFTA technology in the practical context of emergency decision making, but their applications are limited. Although methods considering the possibility distribution of HFLTSs [3, 11, 38] can solve some of these problems, they are not sufficiently accurate for solving PDM problems.

(2) The current consistency measurement and improvement mechanisms are inaccurate and are not universal. The ordinal consistency in [46-48] is based on the preference relation graph, which is complex and difficult to understand. The additive consistency proposed by Zhang $[46,48]$ involves the immature operation rules of PLTSs, which can easily increase the values elements without reason, increasing the complexity of the calculation and causing the distortion of information. Additionally, although researchers $[15,16,19]$ have rebuilt additive consistencies based on different operation rules of PLTSs, their operation is complex and can easily cause information distortion, because the elements of PLTSs must be consistent. Moreover, for improving the consistency, the iterative algorithms in [13, 41, 46-48] must be subjected to tedious multi-round adjustments by continually approaching the completely consistent matrix obtained in advance, which is very complex. Furthermore, the consistency recovery strategies in $[13,41]$ derive hard-to-understand real numbers using transformation functions. For automatic optimization algorithms, the improved consistency models adopted in $[8,9]$ can only ensure that the consistency satisfies the threshold requirements; they cannot ensure the minimum deviation between the adjusted matrix and the original matrix. Although the optimization algorithm in [15] based on the minimum adjustment size can maintain a small information distortion, the number of adjusted elements is large that 
it is difficult to be accepted by decision-makers.

(3) Because of the complexity of the operation rules of PLTSs, it is tedious to aggregate evaluation values using the aggregation operators in $[15,16,21,46-48]$. Moreover, owing to the immature operation rules of PLTSs, it is easy to cause information distortion in the aggregation process. Additionally, the methods for deriving the priority weight in $[8,9,19,27,41]$ need software to solve the programming model, making them inconvenient and time-consuming for decision-makers who are unfamiliar with programming.

To deeply study the PLTSs with missing information and apply them to PLPDM problems, this paper argues that it is necessary to propose a scientific missing-information estimation mechanism and consistency measure and improvement strategy to overcome the shortcomings of the foregoing research. The challenging tasks that must be performed mainly include the following aspects: 1) clearly defining the PLTSs with incomplete information and estimating the missing information of PLTSs reasonably according to PLPRs; 2) adjusting the consistency of the preference relation effectively and quickly while keeping as much original information as possible and as few adjustment elements as possible (additionally, it is worth considering that the adjusted preference relationship can have as little uncertainty as possible); 3) determining which ranking method is the most convenient and effective for PLPDM problems. According to these motivations, the main contributions and highlights of the present paper are summarized as follows.

(1) The concept of InPLTSs presented in [8], i.e., a form of missing information, is reinterpreted, which covers two possible situations of missing information of PLTSs. The expected multiplicative consistency and information uncertainty of InPLPR are defined. Then, a scientific information completion mechanism called the two-stage optimization model based on the optimal consistency and minimum information uncertainty is proposed, which can deal with many forms of missing information flexibly.

(2) The deviation degree of two InPLTRs is introduced, and then a multi-stage consistency-improving optimization model is developed by considering the minimum deviation from the original matrix, the minimum number of adjusted LTs, and the minimum information uncertainty, which can automatically improve the consistency of the InPLPR to an acceptable threshold in a short time and can obtain a correction result that is easily acceptable to decision-makers.

(3) A mathematical optimization model considering priority is constructed on the basis of the expected multiplicative consistency of the InPLPR. By using mathematical method to solve this model, the priority weight can be derived directly by a general mathematical formula, which facilitates the ranking of alternatives.

(4) To deal with PLPDM problems, a decision-making algorithm based on the multi-stage consistency optimization model with an InPLPR is introduced, and a numerical example of selecting excellent postgraduates for a successive master-doctor project is presented to demonstrate the applicability of the proposed algorithm. Furthermore, a validity test and comprehensive comparative analysis are performed to verify the effectiveness and superiority of the proposed algorithm.

\section{B. Paper structure}

The remainder of this paper is organized as follows. In Section 2, auxiliary concepts are introduced. In Section 3, a two-stage optimization process for estimating missing information of InPLPRs is proposed. A decision-making algorithm based on the multi-stage consistency optimization model for the InPLPR is presented in Section 4. In Section 5, a practical case is presented to demonstrate the applicability of the proposed algorithm, and a detailed validity test and comparative analysis are performed to demonstrate 
the reliability and advantages of the proposed algorithm. Finally, conclusions are presented in Section 6 .

\section{Preliminaries}

\subsection{LTSs}

To present the experts' qualitative judgments (rather than quantitative judgments), Zadeh [44] proposed the LVs. First, we review the LVs.

Suppose that $S=\left\{s_{\alpha} \mid \alpha=0,1, \ldots, 2 \tau\right\}$ is a finite and totally ordered discrete LTS with odd cardinality, where $s_{\alpha}$ represents a possible value for an LV; the term must satisfy the following [7, 12]:

(1) The set is ordered: $s_{\alpha}>s_{\beta}$ if and only if $\alpha>\beta$.

(2) There is a negation operator: $\operatorname{Neg}\left(s_{\alpha}\right)=s_{2 \tau-\alpha}$.

Example 1. When $\tau=4$, a set of nine LTs $S$ can be defined as follows: $S=\left\{s_{0}=\right.$ particularly bad, $s_{1}=$ very bad, $s_{2}=$ bad, $s_{3}=$ somewhat bad, $s_{4}=$ medium, $s_{5}=$ somewhat good, $s_{6}=$ good, $s_{7}=$ very good, $s_{8}=$ particularly good $\}$.

To prevent a loss of information in the operation process, Xu [43] extended the discrete LTS $S$ to a continuous LTS $\tilde{S}=\left\{s_{\alpha} \mid 0 \leq \alpha \leq 2 \tau\right\}$, where if $s_{\alpha} \in S, s_{\alpha}$ can be called the original LT; otherwise, $s_{\alpha}$ can be called the virtual LT. In general, the expert utilizes the original LTs to evaluate information, and the virtual LTs can only appear in operations. Regarding the virtual LTs, Tian et al. [29] reported that by constructing the mapping relationship between them and their corresponding semantics, their corresponding fuzzy semantic representation and linguistic syntax can be calculated.

\subsection{Hesitant fuzzy LTSs (HFLTSs) and extended hesitant fuzzy LTSs (EHFLTSs)}

Considering that a human may have difficulty deciding among several LTs in real decision-making, Rodriguez et al. [24] proposed the following concept of the HFLTS.

Definition 1. [24]. Suppose that $S=\left\{s_{\alpha} \mid \alpha=0,1, \ldots, 2 \tau\right\}$ is an LTS; then, the HFLTS $H_{S}$ is an ordered finite subset of consecutive LTs of $S$.

Example 2. Suppose that $S$ is the LTS of Example 1. Then, we have two different HFLTSs: $H_{S}^{1}=\left\{s_{2}=\right.$ bad, $s_{3}=$ somewhat bad $\} \quad$ and $\quad H_{S}^{2}=\left\{s_{6}=\right.$ good, $s_{7}=$ very good, $s_{8}=$ extremely good $\} \quad$ By simplifying the foregoing results, we obtain $H_{S}^{1}=\left\{s_{2}, s_{3}\right\}$ and $H_{S}^{2}=\left\{s_{6}, s_{7}, s_{8}\right\}$.

However, when considering an information representation involving a group, LVs may not be always consecutive [2, 31, 32]. Thus, Wang [31] proposed the EHFLTS, which is a general form of the HFLTS, by enabling the generalized (either consecutive or non-consecutive) LTs.

Definition 2 [31]. Suppose that $S=\left\{s_{\alpha} \mid \alpha=0,1, \ldots, 2 \tau\right\}$ is an LTS; then, the EHFLTS $H_{S}^{E}$ is an ordered finite subset of the LTs of $S$ represented by $H_{S}^{E}=\left\{s_{i} \mid s_{i} \in S\right\}$.

Remark 1. In a mathematical sense, EHFLTSs are from the union of HFLTSs [32]. In group decision-making (GDM), if individual experts provide their evaluation information by using HFLTSs but their priorities (or importance) are completely unknown, an EHFLTS can be obtained via the union of these HFLTSs, which represents the group evaluation. Thus, EHFLTSs can describe complex decision-making situations in a linguistic setting. 


\subsection{PLTSs}

In the case of individuals' hesitation or their disagreement in the group, the experts may prefer one or some of the possible LTs; thus, the different LTs in an HFLTS or EHFLTS may have different support degrees (or importance degrees). Thus, the assessment information in a hesitant fuzzy linguistic environment includes not only several possible LTs but also the corresponding probabilistic information, which represents the support degree. Therefore, according to the concepts of LDAs, PD-HFLTSs, and PHLTSs, Pang et al. [23] introduced the concept of PLTS.

Definition 3. [23]. Suppose that $S=\left\{s_{\alpha} \mid \alpha=0,1, \ldots, 2 \tau\right\}$ is an LTS, where $\tau$ represents a positive integer; then, a PLTS is defined as follows:

$$
L(p)=\left\{L^{k}\left(p^{k}\right) \mid L^{k} \in S, p^{k} \geq 0, k=1,2, \ldots, \# L(p), \sum_{k=1}^{\# L(p)} p^{k} \leq 1\right\},
$$

where $L^{k}\left(p^{k}\right)$ represents the LT $L^{k}$ with a corresponding occurrence probability $p^{k}$, and $\# L(p)$ represents the number of LTs in $L(p)$.

Remark 2. Regardless of whether HFLTSs or EHFLTSs are used to evaluate objects, experts always have a degree of preference for possible hesitant LTs; that is, different LTs may differ with regard to importance. From this perspective, Wang and Hao [33] proposed the proportional 2-tuples; i.e., two symbolic proportions can be assigned to two successive ordinal LTs in an LTS. Subsequently, Zhang and Dong [45] assigned the symbolic proportions to all the LTs and proposed the concept of the LDA. LDA is derived from an expert who has difficultly deciding among several LTs owing to different decision scenarios, which allows these LTs to be non-consecutive. Additionally, $\mathrm{Wu}$ and $\mathrm{Xu}$ [39] proposed the concept of PD-HFLTS on the basis of the possibility of different LTs in an HFLTS. However, Wu and Xu [39] only studied the case where the possibility is uniformly distributed in the LTs. According to the foregoing research, a novel linguistic representation model called the PHLTS was introduced by Chen et al. [2], which can simultaneously consider the hesitant linguistic assessments of individuals and the corresponding proportional information (support degree) of each generalized LT (either consecutive or non-consecutive) in GDM environments. The PHFLTSs and LDAs are mathematically consistent, but the underlying meanings of their possibility (proportion) associated with generalized LTs are different. The PLTS is an extension of the PHLTS that allows the sum of the probabilities of the possible LTs to be $<1$ in particular decision scenarios.

If $\sum_{k=1}^{\# L(p)} p^{k}=1$, the PLTS has the complete information of the probabilistic distribution of all LTs. If $\sum_{k=1}^{\# L(p)} p^{k}<1$, partial ignorance of the probabilistic distribution exists in a PLTS; this situation may arise from insufficient current information or the absence of opinions of decision-making individuals. In particular, $\sum_{k=1}^{\# L(p)} p^{k}=0$ indicates the complete ignorance of the probabilistic distribution in the PLTS.

For $\sum_{k=1}^{\# L(p)} p^{k}<1$, Pang et al. [23] introduced the following normalization method for estimating the ignorance of the probability.

Definition 2 [23]. Suppose that $L(p)$ is a PLTS with $\sum_{l=1}^{\# L(p)} p_{l} \leq 1$. The corresponding PLTS $\bar{L}(p)=\left\{\bar{L}_{l}\left(\bar{p}_{l}\right) \mid l=1,2, \ldots, \# L(p)\right\} \quad$ is called a standardized PLTS, where $\bar{p}_{l}=p_{l} / \sum_{l=1}^{\# L(p)} p_{l}$ for all $l=1,2, \ldots, \# L(p)$.

This is the most primitive and commonly used method for completing information. 


\subsection{Incomplete PLTSs (InPLTSs)}

Usually, when the decision-maker's professional knowledge is limited and the relevant information is insufficient (particularly in some emergency decision-making situations), the occurrence probabilities of some or even all the LTs in PLTSs are difficult to determine. Additionally, in the real GDM environment, some experts may not express their opinions. These situations lead to missing information in PLTSs. Therefore, Gao et al. [8] proposed the concept of the InPLTS.

Definition 3 [8]. Suppose that $S=\left\{s_{\alpha} \mid \alpha=0,1, \ldots, 2 \tau\right\}$ is an LTS, where $\tau$ represents a positive integer. Then, an InPLTS is defined as follows:

$$
L(x)=\left\{L^{k}\left(x^{k}\right) \mid L^{k} \in S, 0 \leq x^{k} \leq 1, k=1,2, \ldots, \# L(x), \sum_{k=1}^{\# L(x)} x^{k} \leq 1\right\},
$$

where $x^{k}$ represents the occurrence probability of $L^{k}$, which can be known or unknown. $\# L(x)$ represents the number of different LTs in $L^{k} . L^{k}\left(x^{k}\right)$ represents the uncertain probabilistic linguistic element (PLE).

Remark 3. For the interpretation of the InPLTS, Gao et al. [8] pointed that the InPLTS is an essential extended form of the LTS; they referred to the $L^{k}\left(x^{k}\right)$ as the set of all InPLEs. If all the occurrence probabilities $\left(x^{k}\right)$ are known and denoted as $p^{k}$, the InPLE can be reduced to a PLE. If all the occurrence probabilities are equal to 1, the InPLE can reduced to a hesitant fuzzy linguistic element (HFLE), which can be taken as a generalized HFLE. We believe that the foregoing explanation is not rigorous or clear. Therefore, we present a systematic and reasonable explanation and classification for InPLTSs in this paper.

The InPLTS is an extended form of the PLTS, which cannot obtain complete probability or LV information, owing to the absence of individuals' opinions in the group or the divergence or hesitance of experts' opinions. It can be divided into the following two cases. (1) When all the occurrence probabilities are known and presented as $p^{k}$ with $\sum_{k=1}^{\# L(x)} p^{k}<1$, the InPLTS is an InPLTS with missing LTs (MLT-InPLTS), e.g., $\left\{s_{1}(0.2), s_{3}(0.5), s_{6}(0.2)\right\}$. This form is common in the traditional PLTSs owing to the absence of some experts' opinions. (2) When the occurrence probabilities are partially unknown and $\# x^{k} \geq 2$, where $\# x^{k}$ represents the number of unknown probabilities, the InPLTS is an InPLTS with a missing probability (MP-InPLTS), e.g., $\left\{s_{1}(0.6), s_{5}\left(x_{2}\right), s_{6}\left(x_{3}\right)\right\}$. When the occurrence probability of only one LT is unknown, it can be obtained as $1-\sum_{k=1}^{\# p^{k}} p^{k}$, where $\# p^{k}$ represents the total number of known probabilities; thus, this type of information does not belong to the category of InPLTSs in the present study.

According to the foregoing classification and Remark 2, we can summarize the relationship between InPLTSs and other existing LTSs involving probability. We only discuss the mathematical representation of the information, because the specific meanings and application scenarios may differ.

(1) When $\sum_{k=1}^{\# L(p)} p^{k}<1$, the PLTS is a typical form of missing LT owing to the absence of some experts' opinions and can be called an MLT-InPLTS.

(2) When missing information of the InPLTS is estimated, that is, the complete PLTS (CPLTS) is obtained, InPLTS can evolve into the information form of the LDA and PHLTS.

(3) When missing information of an InPLTS is estimated and all the LTs are consecutive, the InPLTS 
can evolve into the information form of the PD-HFLTS.

(4) When the probabilities of all the LTs are unknown, the information form of the InPLTS is identical to that of EHFLTSs; if these LTs are consecutive, the information form of the InPLTS is identical to that of HFLTSs.

Therefore, the information model based on the InPLTS can express or evolve into a variety of existing linguistic information models, and its adaptability is extensive and flexible. To facilitate the following study, we present the following definitions.

Definition 4. Let $\bar{L}(x)=\left\{\bar{L}^{k}\left(x^{k}\right) \mid k=1,2, \ldots, \# \bar{L}(x)\right\}$ be an InPLTS. If all the LTs $\left(\bar{L}^{k}\right)$ and their occurrence probabilities $\left(x^{k}\right)$ are complete and known, i.e., $\sum_{k=1}^{\# \bar{L}(x)} x^{k}=1$, this InPLTS is called a CPLTS.

The mathematical forms of CPLTSs are identical to those of LDAs and PHLTSs, but their specific sources and application meanings differ.

Definition 5. Let $L(x)$ be an InPLTS, $\bar{L}(x)=\left\{\bar{L}^{k}\left(x^{k}\right) \mid k=1,2, \ldots, \# \bar{L}(x)\right\}$ be a CPLTS obtained from $L(x)$, and $r^{k}$ be the subscript of $\bar{L}^{k}$. Then, the expected value $E(L(x))$ of $L(x)$ is given as follows:

$$
E(L(x))=\sum_{k=1}^{\# \bar{L}(x)} r^{k} x^{k},
$$

where $E(L(x)) \in[0,2 \tau]$. The $E(L(x))$ can be denoted as $E(\bar{L}(x))$.

\section{Two-stage optimization process to estimate missing information for InPLTR}

\section{based on consistency}

\subsection{InPLPRs}

Because practical decision-making is similar to the process of thinking, the judgment mode of pairwise comparison is easy to accept by experts. The InPLTS is suitable for this type of evaluation, because it allows experts to provide incomplete information under the circumstances of time pressure or insufficient knowledge. Therefore, the preference relation based on the InPLTS can be constructed to solve PDM problems.

Let $A=\left\{a_{1}, a_{2}, \ldots, a_{n}\right\}$ denote a finite set of alternatives. If a group of experts is asked to perform pairwise comparisons utilizing the InPLTS, they can construct a preference relation $R=\left(L(x)_{i j}\right)_{n \times n}$, whose elements $L(x)_{i j}$ represent the priority degree of alternative $a_{i}$ over $a_{j}$. The preference relation with InPLTSs is defined as follows.

Definition 6 [23]. Suppose that $L(x)_{i j}=\left\{L_{i j}^{k}\left(x_{i j}^{k}\right) \mid k=1,2, \ldots, \# L(x)_{i j}\right\}$ is an InPLTS. Then, the matrix $R=\left(L(x)_{i j}\right)_{n \times n}$ is called an InPLPR, where $r_{i j}^{k}$ is the subscript of the LT $L_{i j}^{k}$ and $L(x)_{i j}$ must satisfy the following conditions:

(1) $r_{i j}^{k}+r_{j i}^{k}=2 \tau, x_{i j}^{k}=r_{j i}^{k}, r_{i i}^{k}=\tau, x_{i i}^{k}=1, \# L(x)_{i j}=\# L(x)_{j i} \quad\left(\# L(x)_{i j}\right.$ and $\# L(x)_{j i}$ represent the numbers of possible elements in $L(x)_{i j}$ and $L(x)_{j i}$, respectively);

(2) $r_{i j}^{k}<r_{i j}^{k+1}$ and $r_{j i}^{k}<r_{j i}^{k+1}$, where $i<j, i=1,2, \ldots, n-1, j=2,3, \ldots, n, l=1,2, \ldots, \# L(x)_{i j}-1$.

According to Definition 6, the following conclusions can be drawn:

(1) The InPLTS is the basic element of the InPLPR.

(2) The LTs of the InPLTS in the upper triangular matrix of the InPLPR are increasing; conversely, the 
LTs of InPLTS in the lower triangular matrix of InPLPR are decreasing. Thus, given the elements of the upper triangular matrix, we can obtain the corresponding elements of the lower triangular matrix.

(3) The InPLTSs in the diagonal line of the InPLPR are equal; they are all $s_{\tau}(1)$.

Definition 7. Suppose that $\bar{L}(x)_{i j}=\left\{\bar{L}_{i j, k}\left(x_{i j, k}\right) \mid k=1,2, \ldots, \# \bar{L}(x)_{i j}\right\}$ is a CPLTS; then, the matrix $\bar{R}=\left(\bar{L}(x)_{i j}\right)_{n \times n}$ is called a complete PLPR (CPLPR).

\subsection{Expected multiplicative consistency of InPLPRs}

In the fuzzy preference relation, there is a logical assumption that if $X$ takes precedence over $Y$ and $Y$ takes precedence over $Z, X$ will take precedence over $Z$. Consistency is an important indicator for testing this logical relationship in fuzzy preference relations [4, 22]. However, in some realistic decision-making environments, it is difficult to give a completely consistent preference relation, because of the expert's psychological judgment error. As a generalized linguistic preference relation, InPLPRs also need to be tested for consistency. First, we review the transitive properties of linguistic preference relations.

Suppose that $A=\left(a_{i j}\right)_{n \times n}$ is a linguistic preference relation, where $a_{i j} \in S$. Some transitive properties of $A$ are presented as follows [45].

(1) Weak transitivity: $a_{i j} \geq s_{t}, a_{j h} \geq s_{t} \Rightarrow a_{i h} \geq s_{t}, \forall i, j, h$.

(2) Additive transitivity: $I\left(a_{i h}\right)=I\left(a_{i j}\right)+I\left(a_{j h}\right)-\tau, \forall i, j, h$.

(3) Multiplicative transitivity: $I\left(a_{i j}\right) \times I\left(a_{j h}\right) \times I\left(a_{h i}\right)=I\left(a_{i h}\right) \times I\left(a_{h j}\right) \times I\left(a_{j i}\right)$ if $I\left(a_{i j}\right), \quad I\left(a_{j h}\right)$, $I\left(a_{i h}\right) \notin\{0,2 \tau\}$, where $I\left(a_{i j}\right)$ is the subscript of the LT $a_{i j}$.

Remark 4. In a sense, the additive consistency and the multiplicative consistency are parallel in terms of consistency measures. One of them is based on the difference scale, and the other is based on the ratio scale, and they both satisfy the weak transitivity and some restricted transitivity (max-min transitivity and max-max transitivity) defined in [28]. However, some scholars have found that there are limitations in the additive consistency [17, 40,49]. For example, for an additive consistent linguistic preference relation $R$, let $\tau=3$. If $a_{12}=s_{5}$ and $a_{23}=s_{5}$, according to the formula of the additive transitivity, we obtain $I\left(a_{13}\right)=I\left(a_{12}\right)+I\left(a_{23}\right)-\tau=5+5-3=7$. Thus, $a_{13}=s_{7}$, which is beyond the scope of the original domain and is unreasonable. Although $a_{13}$ can be transformed into the values in $\left[s_{0}, s_{6}\right]$, some preference information is distorted.

Considering that the multiplicative consistency can avoid the limitation of the additive consistency and is inspired by the foregoing multiplicative transitivity, we propose the following expected multiplicative consistency of InPLPRs.

Definition 8. Suppose that $R=\left(L(x)_{i j}\right)_{n \times n}$ is an InPLPR matrix and $\bar{R}=\left(\bar{L}(x)_{i j}\right)_{n \times n}$ is its corresponding CPLPR. Then, $R=\left(L(x)_{i j}\right)_{n \times n}$ is expected to be multiplicatively consistent for all $i, j, h=1,2, \ldots, n, i \neq j \neq h$ and $E\left(\bar{L}(x)_{i j}\right), E\left(\bar{L}(x)_{j h}\right), E\left(\bar{L}(x)_{h i}\right) \notin\{0,2 \tau\} \quad$ if

$$
E\left(\bar{L}(x)_{i j}\right) E\left(\bar{L}(x)_{j h}\right) E\left(\bar{L}(x)_{h i}\right)=E\left(\bar{L}(x)_{j i}\right) E\left(\bar{L}(x)_{i h}\right) E\left(\bar{L}(x)_{h j}\right)
$$

where $E\left(\bar{L}(x)_{i j}\right)$ represents the expected value of the CPLTS $\bar{L}(x)_{i j}$. Hereinafter, for simplicity, $E\left(\bar{L}(x)_{i j}\right)$ is denoted as $\bar{E}_{i j}$. 
By changing the mathematical equation of Eq. (4) and taking logarithms on both sides of the equals sign, the expression of Eq. (4) can be redefined as follows.

Definition 9. Let $R=\left(L(x)_{i j}\right)_{n \times n}$ be an InPLPR matrix and $\bar{R}=\left(\bar{L}(x)_{i j}\right)_{n \times n}$ be its corresponding CPLPR. Then, $R=\left(L(x)_{i j}\right)_{n \times n}$ is expected to be multiplicatively consistent for all $i, j, h=1,2, \ldots, n, i \neq j \neq h$ and $\bar{E}_{i j}, \bar{E}_{j h}, \bar{E}_{i h} \notin\{0,2 \tau\} \quad$ if

$$
\ln \bar{E}_{i j}+\ln \bar{E}_{j h}+\ln \bar{E}_{h i}=\ln \bar{E}_{j i}+\ln \bar{E}_{i h}+\ln \bar{E}_{h j} .
$$

In practice, owing to various complex factors, a completely consistent preference relation is difficult to obtain. For utilizing these preference matrices more efficiently, it is necessary to define a consistency index to measure the level of consistency.

According to Definition 9, an expected multiplicative consistency index for InPLPRs can be defined as follows.

Definition 10. Suppose that $R=\left(L(x)_{i j}\right)_{n \times n}$ is an InPLPR matrix and $\bar{R}=\left(\bar{L}(x)_{i j}\right)_{n \times n}$ is its corresponding CPLPR. Then, the expected multiplicative consistency index of $R$ is defined as follows:

$$
C I(R)=\frac{1}{C_{n}^{3}} \sum_{1 \leq i<j<h \leq n}\left|\ln \bar{E}_{i j}+\ln \bar{E}_{j h}+\ln \bar{E}_{h i}-\ln \bar{E}_{j i}-\ln \bar{E}_{i h}-\ln \bar{E}_{h j}\right| .
$$

Clearly, $C I(R) \geq 0$, and if an InPLPR is expected to be multiplicatively consistent, its consistency index is $C I(R)=0$. Conversely, if the consistency index is $C I(R)=0$, the InPLPR is expected to be completely multiplicatively consistent. A smaller value of $C I(R)$ corresponds to a higher consistency level of the InPLPR (and vice versa).

\subsection{Two-stage optimization process to estimate missing information for InPLPRs}

In the previous subsection, we introduced the InPLPR based on the InPLTSs. To confirm the logical hypothesis of the InPLPR, an expected multiplicative consistency is proposed. However, in the actual decision-making process, owing to the effects of various factors, it is difficult for decision-makers to provide a preference relation with complete information. The key point is that the complete information is the prerequisite for the effective operation of InPLTSs. Therefore, before processing the InPLPRs, we must estimate the missing information.

For the InPLPR, we must deal with two cases of missing information. One case is where the elements of the InPLPR have MLT-InPLTSs. Another case is where the elements of the InPLPR have MP-InPLTSs. The former case is common for traditional PLTSs. According to the review of Mi et al. [20], thus far, the normalization of PLTSs can be divided into five categories according to the assignment of the unknown probabilities: (1) the average assignment, (2) full-set assignment, (3) pow-set assignment, (4) envelope assignment, and (5) attitude-based assignment. They all have defects. Although methods (1) and (4) are relatively simple and fast, they are inappropriate and imprudent and cannot reflect the nature of the decision-makers' hesitation. Therefore, such methods easily cause information distortion. Methods (2) and (3) can easily result in too many elements in PLTSs, which is not convenient for the operation of PLTSs. Method (4) can easily cause inconsistent mathematical expressions of element terms, which is not conducive to the later operation. For the traditional PLTSs with the missing probability, from the perspective of individual judgment, we believe that the experts must have an uncertain cognition in their evaluation result. In this uncertain cognition, they are unsure which LT or LTs should be selected. From the perspective of collective judgment, such information represents the absence of some expert opinions. 
We believe that in these decision-making contexts, the unknown probability may occur in LTs ranging from $s_{0}$ to $s_{2 \tau}$. Thus, we assume that the missing probability $\left(1-\sum_{k=1}^{\# L(x)_{i j}} p_{i j}^{k}\right)$ only exists in a virtual LT $s_{r}$ that integrates all possible scenarios, and the range of $r$ is $0 \leq r \leq 2 \tau$. For satisfying the needs of the decision models or the requirements of the decision-makers, the missing LT must be in the original discrete domain of the LTS. In this case, we can let $r$ be an integer in the range of $[0,2 \tau]$.

\section{Stage 1 Optimization}

Consistency is a key index for judging the rationality of the preference relation. Of course, we hope that the preference relation needed for decision-making can achieve the highest possible level of consistency. To estimate the incomplete information, we use the expected multiplicative consistency to construct an optimization model. When $C I(R)=0$, the InPLPR is expected to be multiplicatively consistent. However, this situation is difficult to achieve in reality. Consequently, we can minimize the $C I(R)$; a smaller $C I(R)$ indicates that the InPLPR is closer to a completely consistent InPLPR and that the preference evaluation is more accurate. For the convenience of modeling, we define the following: $O=\left\{(i, j) \mid L(x)_{i j}\right.$ is CPLTS $\}, P=\left\{(i, j) \mid L(x)_{i j}\right.$ is MP-InPLTS $\}$, and $Q=\left\{(i, j) \mid L(x)_{i j}\right.$ is MLT-InPLTS $\}$ Then, the corresponding expected values of InPLTSs from the InPLPR can be obtained, as follows:

$$
\bar{E}_{i j}=\left\{\begin{array}{ll}
\sum_{k=1}^{\# L(x)_{i j}} r_{i j}^{k} p_{i j}^{k}, & (i, j) \in O \\
\sum_{k=1}^{\# L(x)_{i j}} r_{i j}^{k} x_{i j}^{k}, & (i, j) \in P . \\
\sum_{k=1}^{\# L(x)_{i j}+1} r_{i j}^{k} x_{i j}^{k}, & (i, j) \in Q .
\end{array} .\right.
$$

We construct the following optimization model to obtain missing information:

$$
\begin{aligned}
& \min C I=\frac{1}{C_{n}^{3}} \sum_{1 \leq i<j<h \leq n}\left|\ln \bar{E}_{i j}+\ln \bar{E}_{j h}+\ln \bar{E}_{h i}-\ln \bar{E}_{j i}-\ln \bar{E}_{i h}-\ln \bar{E}_{h j}\right| \\
& =\frac{1}{C_{n}^{3}} \sum_{1 \leq i<j<h \leq n}\left|\begin{array}{l}
\ln \sum_{k=1}^{\# \bar{L}(x)_{i j}} r_{i j}^{k} x_{i j}^{k}+\ln \sum_{k=1}^{\# \bar{L}(x)_{j k}} r_{j h}^{k} x_{j h}^{k}+\ln \sum_{k=1}^{\# \bar{L}(x)_{h i}} r_{h i}^{k} x_{h i}^{k} \\
-\ln \sum_{k=1}^{\# \bar{L}(x)_{j i}} r_{j i}^{k} x_{j i}^{k}-\ln \sum_{k=1}^{\# \bar{L}(x)_{i h}} r_{i h}^{k} x_{i h}^{k}-\ln \sum_{k=1}^{\# \bar{L}(x)_{h j}} r_{h j}^{k} x_{h j}^{k}
\end{array}\right| \\
& \text { s.t. }\left\{\begin{array}{lr}
\# \bar{L}(x)_{i j}=\# L(x)_{i j}, & (i, j) \in O \text { or }(i, j) \in P \\
\# \bar{L}(x)_{i j}=\# L(x)_{i j}+1, & (i, j) \in Q \\
0 \leq r_{i j}^{\# L(x)_{i j}+1} \leq 2 \tau, & (i, j) \in Q \\
\sum_{k=1}^{\# \bar{L}(x)_{i j}} x_{i j}^{k}=1, & \\
\sigma \leq x_{i j}^{k} \leq 1, x_{i j}^{k}=x_{j i}^{k}, & k=1,2, \ldots, \# \bar{L}(x)_{i j} \\
r_{i j}^{k}+r_{j i}^{k}=2 \tau, & k=1,2, \ldots, \# \bar{L}(x)_{i j},
\end{array}\right.
\end{aligned}
$$

where the objective function ensures that the level of consistency of the preference matrix is highest, i.e., that the deviation is minimized. The second and third constraints deals with the MLT-InPLTSs. the fourth to sixth constraints ensure that $\bar{L}(x)_{i j}$ is a CPLTS. $r_{i j}^{\# L(x)_{i j}+1}$ and $x_{i j}^{k}$ are decision variables.

$$
\begin{aligned}
& \text { To solve the model (8) more easily , we define } \\
& \varepsilon_{i j h}=\ln \sum_{k=1}^{\# \bar{L}(x)_{i j}} r_{i j}^{k} x_{i j}^{k}+\ln \sum_{k=1}^{\# \bar{L}(x)_{j k}} r_{j h}^{k} x_{j h}^{k}+\ln \sum_{k=1}^{\# \bar{L}(x)_{h i}} r_{h i}^{k} x_{h i}^{k}-\ln \sum_{k=1}^{\# \bar{L}(x)_{j i}} r_{j i}^{k} x_{j i}^{k}-\ln \sum_{k=1}^{\# \bar{L}(x)_{i h}} r_{i h}^{k} x_{i h}^{k}-\ln \sum_{k=1}^{\# \bar{L}(x)_{h j}} r_{h j}^{k} x_{h j}^{k} \\
& \text {, } \varepsilon_{i j h}^{+}=\left(\left|\varepsilon_{i j h}\right|+\varepsilon_{i j h}\right) / 2 \text {, and } \varepsilon_{i j h}^{-}=\left(\left|\varepsilon_{i j h}\right|-\varepsilon_{i j h}\right) / 2 \text {; thus, }\left|\varepsilon_{i j h}\right|=\varepsilon_{i j h}^{+}+\varepsilon_{i j h}^{-} \text {and } \varepsilon_{i j h}=\varepsilon_{i j h}^{+}-\varepsilon_{i j h}^{-} \text {. Moreover, }
\end{aligned}
$$
according to Definition 6, model (8) can be simplified by applying its upper triangular components. Hence, model (8) can be transformed into the following: 


$$
\begin{aligned}
& \min C I=\frac{1}{C_{n}^{3}} \sum_{1 \leq i<j<h \leq n}\left(\varepsilon_{i j h}^{+}+\varepsilon_{i j h}^{-}\right) \\
& s . t .\left\{\begin{array}{lc}
\ln \sum_{k=1}^{\# \bar{L}(x)_{i j}} r_{i j}^{k} x_{i j}^{k}+\ln \sum_{k=1}^{\# \bar{L}(x)_{j k}} r_{j h}^{k} x_{j h}^{k}+\ln \sum_{k=1}^{\# \bar{L}\left(x x_{i h}\right.}\left(2 \tau-r_{i h}^{k}\right) x_{i h}^{k}-\ln \sum_{k=1}^{\# \bar{L}(x)_{i j}}\left(2 \tau-r_{i j}^{k}\right) x_{i j}^{k} \\
-\ln \sum_{k=1}^{\# \bar{L}(x)_{i j}} r_{i h}^{k} x_{i h}^{k}-\ln \sum_{k=1}^{\# \bar{L}(x)_{j h}}\left(2 \tau-r_{j h}^{k}\right) x_{j h}^{k}-\varepsilon_{i j h}^{+}+\varepsilon_{i j h}^{-}=0, \quad 1 \leq i<j<h \leq n \\
\# \bar{L}(x)_{i j}=\# L(x)_{i j} \quad(i, j) \in O \text { or }(i, j) \in P \\
\# \bar{L}(x)_{i j}=\# L(x)_{i j}+1, & (i, j) \in Q \\
0 \leq r_{i j, \# L(x)_{i j}+1} \leq 2 \tau, & (i, j) \in Q \\
\sum_{k=1}^{\# \bar{L}(x)_{i j}} x_{i j}^{k}=1, & i=1,2, \ldots, n-1 ; j=i+1, i+2, \ldots, n \\
\varepsilon_{i j h}^{+}, \varepsilon_{i j h}^{-} \geq 0, \varepsilon_{i j h}^{+} \varepsilon_{i j h}^{-}=0 & 1 \leq i<j<h \leq n .
\end{array}\right.
\end{aligned}
$$

Model (9) does not ensure the existence of an optimal solution. For this case, we adopt the following method.

Studies have indicated that models similar to model (9) may produce virtual LTs that do not exist in the original LTs, making these results difficult for decision-makers to understand. Therefore, integer programming has been widely studied. In fact, virtual LTs are not difficult to understand, because they can provide the corresponding fuzzy semantic representation and linguistic syntax through relevant mapping. Additionally, we believe that in some emergency decision-making situations where expert feedback is not required, virtual LTs can help to obtain more accurate evaluation results than original LTs. However, if practical problems cannot be solved using model (9), a corresponding integer programming model can be used:

$$
\begin{aligned}
& \min C I=\frac{1}{C_{n}^{3}} \sum_{1 \leq i<j<h \leq n}\left(\varepsilon_{i j h}^{+}+\varepsilon_{i j h}^{-}\right)
\end{aligned}
$$

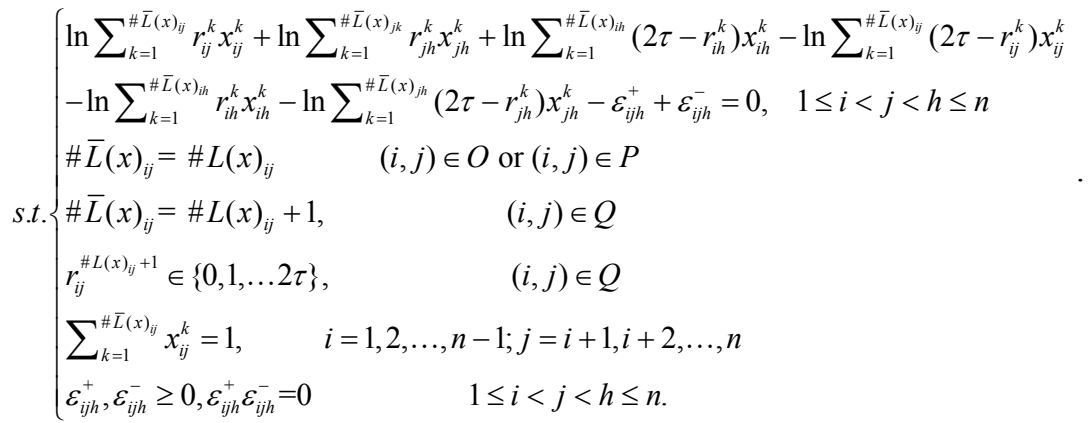

\section{Stage 2 Optimization}

Considering that there may be at least one pair of solutions in models (8) and (10), although they are all feasible solutions, for adapting to the actual decision-making problem, we adopt the following method to obtain the final solution.

PLTSs arise from the uncertainty of information. The Shannon's entropy [5] can accurately reflect the uncertainty of this information according to the occurrence probabilities of variables (LTs). A greater uncertainty of the variables corresponds to a larger entropy and a larger amount of information needed to be made clear. Therefore, to better mine the information uncertainty in PLTSs, we present the following definition.

Definition 11. Let $\bar{L}(x)=\left\{\bar{L}^{k}\left(x^{k}\right) \mid k=1,2, \ldots, \# \bar{L}(x)\right\}$ be a CPLTS and $x^{k}$ be the corresponding occurrence probability of the possible LT $\bar{L}^{k}$. Then, the information uncertainty of $\bar{L}(x)$ is given as follows: 


$$
u(\bar{L}(x))=-\sum_{k=1}^{\# \bar{L}(x)} x^{k} \log _{2} x^{k} .
$$

Clearly, a smaller uncertainty of the CPLTS corresponds to a smaller entropy, a higher accuracy of the information, and a higher accuracy of the evaluation results. Thus, if there are multiple optimal solutions in model (9) or (10), for identifying the optimal solution, the optimization models (12) and (13) based on the minimum uncertainty (minimum information entropy) can be constructed.

$$
\begin{aligned}
& \min u=-\frac{1}{z} \sum_{r=1}^{z} \sum_{k=1}^{\# \bar{L}(x)_{i j, r}} x_{i j, r}^{k} \log _{2} x_{i j, r}^{k} . \\
& \text { s.t. }\left\{\begin{array}{l}
\frac{1}{C_{n}^{3}} \sum_{1 \leq i<j<h \leq n}\left(\varepsilon_{i j h}^{+}+\varepsilon_{i j h}^{-}\right)=C I_{*} \\
\text { All other constraints in model (9). }
\end{array}\right.
\end{aligned}
$$

Here, $\bar{L}(x)_{i j, r}(r=1,2, \ldots z)$ represents the $r^{\text {th }}$ InPLTS that must be estimated for the missing probability. The objective function ensures that the total uncertainty of the estimated CPLTSs is minimized. $C I_{*}$ represents the optimal value for model (9), and the other constraints are identical to those for model (9).

$$
\begin{aligned}
& \min u=-\frac{1}{z} \sum_{r=1}^{z} \sum_{k=1}^{\# \bar{L}(x)_{i, r}, r} x_{i j, r}^{k} \log _{2} x_{i j, r}^{k} . \\
& \text { s.t. }\left\{\begin{array}{l}
\frac{1}{C_{n}^{3}} \sum_{1 \leq i<j<h \leq n}\left(\varepsilon_{i j h}^{+}+\varepsilon_{i j h}^{-}\right)=C I_{*} \\
\text { All other constraints in model (10), }
\end{array}\right.
\end{aligned}
$$

Here, $C I_{*}$ represents the optimal value for model (10), and the other constraints are identical to those for model (10)

Then, by solving model (12) or (13) using a software package (LINGO or MATLAB), a CPLPR with complete information can be obtained.

Remark 5. When dealing with InPLPRs, which only contain the incomplete information of MLT-InPLTSs, e.g., the traditional PLPRs, it is considered that only the missing LTs must be estimated. In this case, the information uncertainty of the estimated CPLTSs needs to be measured in a different way. In the actual decision-making situation, the existence of multiple LTs in PLTSs or HFLTSs is often due to the lack of concentration of opinions and reflects the uncertainty of information. Therefore, a higher concentration of these LTs is better for decision-makers. From this perspective, the uncertainty of the information can be measured according to the discreteness of the LTs.

To measure the uncertainty of $\bar{L}(x)$, we employ the statistical concept of variance to measure the degree of dispersion of LTs in CPLTSs. Thus, the specific uncertainty measurement of $\bar{L}(x)$ can be calculated as follows:

$$
u^{\prime}(\bar{L}(x))=\sum_{k=1}^{\# \bar{L}(x)}\left(r_{i j}^{k}-\frac{1}{\# \bar{L}(x)} \sum_{k=1}^{\# \bar{L}(x)} r_{i j}^{k}\right)^{2} x_{i j}^{k} .
$$

According to formula (14), models (12) and (13) can be substituted into the following two models:

$$
\begin{aligned}
& \min u^{\prime}=\frac{1}{z} \sum_{r=1}^{z} \sum_{k=1}^{\# \bar{L}(x)_{i j, r}}\left(r_{i j, r}^{k}-\frac{1}{\# \bar{L}(x)} \sum_{k=1}^{\# \bar{L}(x)_{i j, r}} r_{i j, r}^{k}\right)^{2} x_{i j, r}^{k} \\
& \text { s.t. }\left\{\begin{array}{l}
\frac{1}{C_{n}^{3}} \sum_{1 \leq i<j<h \leq n}\left(\varepsilon_{i j h}^{+}+\varepsilon_{i j h}^{-}\right)=C I_{*} \\
\text { All other constraints in model (9), }
\end{array}\right.
\end{aligned}
$$

and 
where $\bar{L}(x)_{i j, r}(r=1,2, \ldots z)$ represents the $r^{\text {th }}$ InPLTS that must be estimated for the missing LT.

Models (15) and (16) are only two alternatives. When there are two forms of incomplete information of InPLTSs in the InPLPRs, the use of model (13) or (14) is prioritized for estimating missing information in Stage 2.

Example 3. Assume that there are four alternatives $\left(A_{1}, A_{2}, A_{3}\right.$, and $\left.A_{4}\right)$ for ranking selection. Considering the urgency of the decision-making and the lack of knowledge, experts decide to use an InPLPR to evaluate these alternatives. According to the LTS $S=\left\{s_{0}=\right.$ particularly bad, $s_{1}=$ very bad, $s_{2}=$ bad, $s_{3}=$ somewhat bad, $s_{4}=$ medium, $s_{5}=$ somewhat good, $s_{6}=$ good, $s_{7}=$ very good, $s_{8}=$ particularly good $\}$, the following InPLPR matrix $R=\left(L(x)_{i j}\right)_{3 \times 3}$ is provided.

$$
R=\left[\begin{array}{lccc}
\left\{s_{4}(1)\right\} & \left\{s_{4}\left(x_{12,1}\right), s_{5}(0.4), s_{6}\left(x_{12,3}\right)\right\} & \left\{s_{5}(1)\right\} & \left\{s_{4}\left(x_{14,1}\right), s_{6}\left(x_{14,2}\right)\right\} \\
\left\{s_{4}\left(x_{21,1}\right), s_{2}(0.4), s_{1}\left(x_{21,3}\right)\right\}\left\{s_{4}(1)\right\} & \left\{s_{3}(1)\right\} & \left\{s_{2}(0.6), s_{3}(0.2)\right\} \\
\left\{s_{3}(1)\right\} & \left\{s_{5}(1)\right\} & \left\{s_{4}(1)\right\} & \left\{s_{3}(0.6), s_{5}(0.4)\right\} \\
\left\{s_{4}\left(x_{41,1}\right), s_{2}\left(x_{41,2}\right)\right\} & \left\{s_{2}(0.6), s_{3}(0.2)\right\} & \left\{s_{5}(0.6), s_{3}(0.4)\right\}\left\{s_{4}(1)\right\}
\end{array}\right]
$$

For InPLTS $L(x)_{12}$ and $L(x)_{14}$ in $R$, the occurrence probabilities of the LTs are partially missing or completely missing; that is, they are MP-InPLTSs. For InPLTS $L(x)_{24}$, the occurrence probabilities of all possible LTs are known, but their sum is $<1$. Thus, $L(x)_{24}$ is an MLT-InPLTS that lacks a virtual LT with a probability of 0.1 .

We employ the proposed two-stage model to estimate the missing information. In the first stage, by solving this InPLPR matrix using model (9) (LINGO 11.0 was employed to solve all the programming models in this study), we obtain the following CPLPR matrix based on the optimal solution:

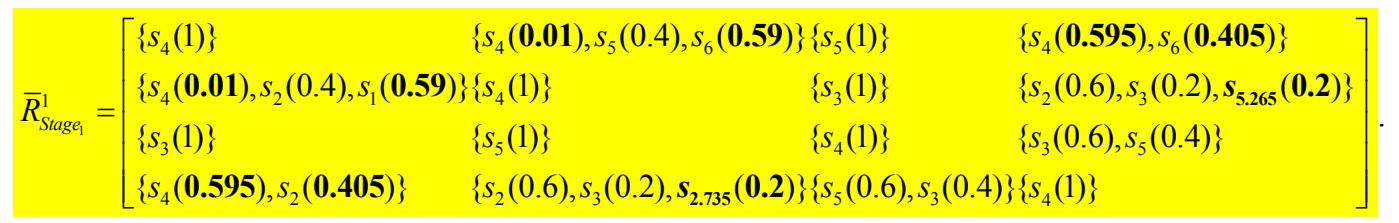

However, as it is unclear whether the uncertainty of the estimated CPLPR is minimal, the second-stage model (12) is implemented, and the following CPLPR based on the optimal solution is obtained:

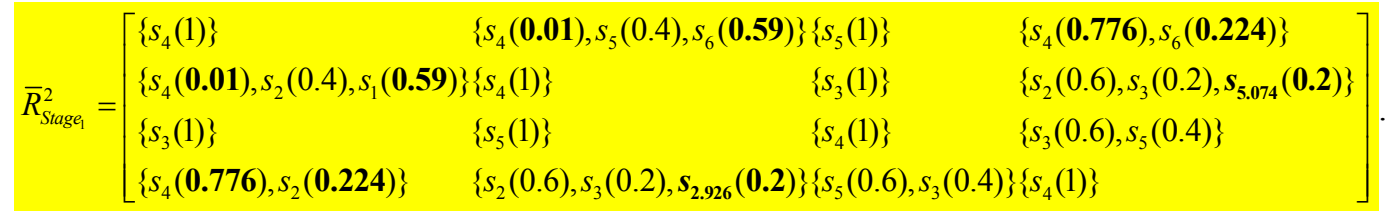

As indicated by the foregoing example, for the missing-information estimation of InPLPRs, an ideal solution can be obtained by using the proposed two-stage optimization model. 


\section{Decision-making algorithm based on multi-stage consistency optimization model with InPLPR}

\subsection{Multi-stage consistency-improving optimization model for InPLPR}

There are two main types of consistency adjustment strategies. The first involves local feedback adjustment with the participation of decision-makers. The matrix with acceptable consistency obtained via this adjustment method can yield the optimal recognition of decision-makers. However, this method is time-consuming and may lead to the excessive adjustment of elements. The second type of strategy involves an automatic improvement algorithm, e.g., automatic iterative adjustment or automatic optimization adjustment. The automatic iterative algorithm also belongs to the category of local adjustment, as the adjusted elements must be identified in advance. The automatic optimization algorithm can adjust the matrix elements in the form of global optimization in a short time and can satisfy the certain target requirements, such as the minimum adjustment size (minimum information distortion). However, it has shortcomings, e.g., the adjusted matrix may not be satisfied and accepted by decision-makers. For improving the time efficiency and decision accuracy, we tend to select the automatic optimization adjustment algorithm in this study. In particular, for dealing with urgent decision-making problems, we must respect and make full use of the original information provided by experts instead of asking them to revise their opinions round-by-round. Additionally, studies have been performed on the feedback adjustment method based on the optimization results, which can balance the decision-making time (or complexity) and the satisfaction of decision-makers. The development of such a method based on the InPLPR is left for future research.

In practical decision-making, it is not easy to provide a completely consistent preference relation, owing to various factors. However, a preference relation with insufficient consistency leads to unreasonable evaluation results. Therefore, to deal with an InPLPR with unacceptable consistency, it is necessary to develop a mechanism to improve the consistency effectively. Generally, there are two primary factors to be considered in the improved mechanism: the time consumption and information distortion. So, the following research will be performed.

Let $R=\left(L(x)_{i j}\right)_{n \times n}$ be an InPLPR matrix and $\bar{R}=\left(\bar{L}(x)_{i j}\right)_{n \times n}$ be its corresponding CPLPR matrix. $\overline{C I}$ is a predetermined threshold of the expected multiplicative consistency index, $\overline{C I} \geq 0$. If $C I(\bar{R}) \leq \overline{C I}, R$ can be viewed as an acceptable consistent InPLPR; otherwise, $R$ is viewed as an InPLPR with unacceptable consistency. The value of $\overline{C I}$ is usually obtained in a variety of ways, such as the actual situation of decision-making, the preference habits and requirements of decision-makers, or simulation. In this study, through small-scale simulation experiments, the acceptable consistent matrices were generated randomly within the acceptable error range. We find that the consistency is related to the magnitudes of the LTs used and the standard deviation allowed, but the result is generally $<0.15$. To have more stringent requirements for consistency and make the matrix have better logical relations, $\overline{C I}$ is set as 0.1 . Such problems will be studied further in the future.

To measure the distortion of information, the deviation of two CPLTSs with the same number of LT elements can be defined.

Definition 12. Let $\bar{L}(x)_{1}=\left\{\bar{L}_{1}^{k}\left(x_{1}^{k}\right) \mid k=1,2, \ldots, \# \bar{L}(x)_{1}\right\}$ and $\bar{L}(x)_{2}=\left\{\bar{L}_{2}^{k}\left(x_{2}^{k}\right) \mid k=1,2, \ldots, \# \bar{L}(x)_{2}\right\}$ be two CPLTSs. Then, the deviation degree between them can be defined as follows: 


$$
d\left(L(x)_{1}, L(x)_{2}\right)=\frac{1}{4 \tau} \sum_{k=1}^{\# L(x)} \frac{1}{\# L(x)}\left|r_{1}^{k}-r_{2}^{k}\right|+\frac{1}{4 \tau}\left|\bar{E}_{1}-\bar{E}_{2}\right|,
$$

where $\# \bar{L}(x)_{1}=\# \bar{L}(x)_{2}=\# L(x) ; \quad r_{1}^{k}$ and $r_{2}^{k}$ represent the subscripts of LTs $\bar{L}_{1}^{k}$ and $\bar{L}_{2}^{k}$, respectively; and $\bar{E}_{1}$ and $\bar{E}_{2}$ represent the expected values of CPLTSs $\bar{L}(x)_{1}$ and $\bar{L}(x)_{2}$, respectively. Clearly, $0 \leq d\left(\bar{L}(x)_{1}, \bar{L}(x)_{2}\right) \leq 1$, and $d\left(\bar{L}(x)_{1}, \bar{L}(x)_{2}\right)=d\left(\bar{L}(x)_{2}, \bar{L}(x)_{1}\right)$. Moreover, a smaller value of $d\left(\bar{L}(x)_{1}, \bar{L}(x)_{2}\right)$ indicates that $\bar{L}(x)_{1}$ is closer to $\bar{L}(x)_{2} \cdot d\left(\bar{L}(x)_{1}, \bar{L}(x)_{2}\right)=0$ if and only if $\bar{L}(x)_{1}=\bar{L}(x)_{2}$.

According to Definition 12, we can obtain the deviation of two CPLPRs $\bar{R}_{1}$ and $\bar{R}_{2}$, where each corresponding element has the same number of LTs.

Definition 13. Let $\bar{R}_{1}=\left(\bar{L}_{1}(x)_{i j}\right)_{n \times n}$ and $\bar{R}_{2}=\left(\bar{L}_{2}(x)_{i j}\right)_{n \times n}$ be two CPLPRs. The corresponding elements of the two CPLPRs have the same number of LTs; that is, $\# \bar{L}_{1}(x)_{i j}=\# \bar{L}_{2}(x)_{i j}=\# \bar{L}(x)_{i j}$. Then, the deviation degree between $\bar{R}_{1}$ and $\bar{R}_{2}$ can be defined as follows:

$$
d\left(\bar{R}_{1}, \bar{R}_{2}\right)=\frac{1}{2 \tau n(n-1)} \sum_{i=1}^{n-1} \sum_{j=i+1}^{n} \sum_{k=1}^{\# \bar{L}(x)_{i j}} \frac{1}{\# \bar{L}(x)_{i j}}\left|r_{i j, 1}^{k}-r_{i j, 2}^{k}\right|+\frac{1}{2 \tau n(n-1)} \sum_{i=1}^{n-1} \sum_{j=i+1}^{n}\left|\bar{E}_{i j}^{1}-\bar{E}_{i j}^{2}\right|,
$$

where $r_{i j, 1}^{k}$ and $r_{i j, 2}^{k}$ represent the subscripts of LTs $\bar{L}_{i j, 1}^{k}$ and $\bar{L}_{i j, 2}^{k}$, respectively. $\bar{E}_{i j}^{1}$ and $\bar{E}_{i j}^{2}$ represent the expected values of CPLTSs $\bar{L}_{1}(x)_{i j}$ and $\bar{L}_{2}(x)_{i j}$, respectively.

\section{Stage 1 Optimization}

Let $R=\left(L(x)_{i j}\right)_{n \times n}$ be an InPLPR and $\bar{R}=\left(\bar{L}(x)_{i j}\right)_{n \times n}$ be its corresponding CPLPR obtained from model (12) or (13). If $C I(\bar{R})>\overline{C I}, \bar{R}$ is a CPLPR matrix with unacceptable consistency. To obtain an acceptable CPLPR $\tilde{R}$ by maximally retaining the original preference information of CPLPR $\bar{R}$, an optimization model is constructed by minimizing the deviation between the original $\bar{R}$ and the adjusted $\tilde{R}$ under the condition that $\tilde{R}$ satisfies acceptable consistency, that is,

$$
\begin{aligned}
& \min d(\bar{R}, \tilde{R}) \\
& \text { s.t. }\left\{\begin{array}{l}
C I(\tilde{R}) \leq \overline{C I} \\
\tilde{R} \text { is a CPLPR. }
\end{array}\right.
\end{aligned}
$$

Let $\# \bar{L}(x)_{i j}=\# \tilde{L}(x)_{i j}=\# L_{i j}$ in this subsection. According to Eq. (18), we have

$$
d(\bar{R}, \tilde{R})=\frac{1}{2 \tau n(n-1)} \sum_{i=1}^{n-1} \sum_{j=i+1}^{n} \sum_{k=1}^{\# L_{i j}} \frac{1}{\# L_{i j}}\left|r_{i j}^{k}-\tilde{r}_{i j}^{k}\right|+\frac{1}{2 \tau n(n-1)} \sum_{i=1}^{n-1} \sum_{j=i+1}^{n}\left|\bar{E}_{i j}-\tilde{E}_{i j}\right| .
$$

Let $C I(\tilde{R})=\frac{1}{3 C_{n}^{3} \ln 2 \tau} \sum_{i=1}^{n-1} \sum_{j=i+1}^{n}\left|\ln \tilde{E}_{i j}+\ln \tilde{E}_{j h}+\ln \tilde{E}_{h i}-\ln \tilde{E}_{j i}-\ln \tilde{E}_{i h}-\ln \tilde{E}_{h j}\right| \quad$ be the consistency index of $\tilde{R}$. Moreover, because $\tilde{R}$ is a CPLPR, according to Definition 6, the following model is constructed by applying the upper triangular components of $\tilde{R}$ : 
where the objective function minimizes the deviation of the adjusted matrix from the original matrix. The first constraint ensures that the adjusted matrix $\tilde{R}$ is a CPLPR with acceptable consistency. The other constraint ensures that $\tilde{R}$ is a CPLPR. $\tilde{r}_{i j}^{k}$ and $\tilde{x}_{i j}^{k}\left(i, j=1,2, \ldots, n, i \neq j ; \quad k=1,2, \ldots, \# L_{i j}\right)$ are decision variables.

To better solve model (20), we define $\tilde{\varepsilon}_{i j}^{k}=r_{i j}^{k}-\tilde{r}_{i j}^{k}, \tilde{\varepsilon}_{i j}^{k+}=\left(\left|\tilde{\varepsilon}_{i j}^{k}\right|+\tilde{\varepsilon}_{i j}^{k}\right) / 2$, and $\tilde{\varepsilon}_{i j}^{k-}=\left(\left|\tilde{\varepsilon}_{i j}^{k}\right|-\tilde{\varepsilon}_{i j}^{k}\right) / 2$; thus, $\left|\tilde{\varepsilon}_{i j}^{k}\right|=\tilde{\varepsilon}_{i j}^{k+}+\tilde{\varepsilon}_{i j}^{k-}$ and $\tilde{\varepsilon}_{i j}^{k}=\tilde{\varepsilon}_{i j}^{k+}-\tilde{\varepsilon}_{i j}^{k-}$. Analogously, we define $\tilde{\varphi}_{i j}=\bar{E}_{i j}-\tilde{E}_{i j}, \tilde{\varphi}_{i j}^{+}=\left(\left|\tilde{\varphi}_{i j}\right|+\tilde{\varphi}_{i j}\right) / 2$, and $\tilde{\varphi}_{i j}^{-}=\left(\left|\tilde{\varphi}_{i j}\right|-\tilde{\varphi}_{i j}\right) / 2$; thus, $\left|\tilde{\varphi}_{i j}\right|=\tilde{\varphi}_{i j}^{+}+\tilde{\varphi}_{i j}^{-}$and $\tilde{\varphi}_{i j}=\tilde{\varphi}_{i j}^{+}-\tilde{\varphi}_{i j}^{-}$. We define $\tilde{\delta}_{i j h}=\ln \sum_{k=1}^{\# L_{i j}} \tilde{r}_{i j}^{k} \tilde{x}_{i j}^{k}+\ln \sum_{k=1}^{\# L_{j k}} \tilde{r}_{j h}^{k} \tilde{x}_{j h}^{k}+$ $\ln \sum_{k=1}^{\# L_{h i}}\left(2 \tau-\tilde{r}_{i h}^{k}\right) \tilde{x}_{i h}^{k}-\ln \sum_{k=1}^{\# L_{j i}}\left(2 \tau-\tilde{r}_{i j}^{k}\right) \tilde{x}_{i j}^{k}-\ln \sum_{k=1}^{\# L_{i k}} \tilde{r}_{i h}^{k} \tilde{x}_{i h}^{k}-\ln \sum_{k=1}^{\# L_{k j}}\left(2 \tau-\tilde{r}_{j h}^{k}\right) \tilde{x}_{j h}^{k} \quad, \quad \tilde{\delta}_{i j h}^{+}=\left(\left|\tilde{\delta}_{i j h}\right|+\tilde{\delta}_{i j h}\right) / 2 \quad, \quad$ and $\tilde{\delta}_{i j h}^{-}=\left(\left|\tilde{\delta}_{i j h}\right|-\tilde{\delta}_{i j h}\right) / 2$; thus, $\left|\tilde{\delta}_{i j h}\right|=\tilde{\delta}_{i j h}^{+}+\tilde{\delta}_{i j h}^{-}$and $\tilde{\delta}_{i j h}=\tilde{\delta}_{i j h}^{+}-\tilde{\delta}_{i j h}^{-}$. Hence, model (20) is transformed into the following:

$$
\begin{aligned}
& \min d=\frac{1}{2 \tau n(n-1)} \sum_{i=1}^{n-1} \sum_{j=i+1}^{n} \sum_{k=1}^{\# L_{i j}} \frac{1}{\# L_{i j}}\left(\tilde{\varepsilon}_{i j}^{k+}+\tilde{\varepsilon}_{i j}^{k-}\right)+\frac{1}{2 \tau n(n-1)} \sum_{i=1}^{n-1} \sum_{j=i+1}^{n}\left(\tilde{\varphi}_{i j}^{+}+\tilde{\varphi}_{i j}^{-}\right) \\
& s . t .\left\{\begin{array}{l}
r_{i j}^{k}-\tilde{r}_{i j}^{k}-\tilde{\varepsilon}_{i j}^{k+}+\tilde{\varepsilon}_{i j}^{k-}=0, \quad i=1,2, \ldots, n-1 ; j=i+1, i+2, \ldots n ; k=1,2, \ldots, \# L_{i j} \\
\sum_{k=1}^{\# L_{i j}} r_{i j}^{k} x_{i j}^{k}-\sum_{k=1}^{\# L_{i j}} \tilde{r}_{i j}^{k} \tilde{x}_{i j}^{k}-\tilde{\varphi}_{i j}^{+}+\tilde{\varphi}_{i j}^{-}=0, i=1,2, \ldots, n-1 ; j=i+1, i+2, \ldots n ; k=1,2, \ldots, \# L_{i j} \\
\frac{1}{C_{n}^{3}} \sum_{1 \leq i<j<h \leq n}\left(\tilde{\delta}_{i j h}^{+}+\tilde{\delta}_{i j h}^{-}\right) \leq \overline{C I}, \\
\ln \sum_{k=1}^{\# L_{i j}} \tilde{r}_{i j}^{k} \tilde{x}_{i j}^{k}+\ln \sum_{k=1}^{\# L_{j k}} \tilde{r}_{j h}^{k} \tilde{x}_{j h}^{k}+\ln \sum_{k=1}^{\# L_{i j}} \tilde{r}_{i h}^{k} \tilde{x}_{i h}^{k}-\ln \sum_{k=1}^{\# L_{l i}}\left(2 \tau-\tilde{r}_{j h}^{k}\right) \tilde{x}_{j h}^{k}-\tilde{\delta}_{i j h}^{+}+\tilde{\delta}_{i j h}^{-}=0, \quad 1 \leq i<j<h \leq n \\
0 \leq \tilde{r}_{i j}^{k} \leq 2 \tau \text { with } \tilde{r}_{i j}^{k}<\tilde{r}_{i j}^{k+1}, \quad i=1,2, \ldots, n-1 ; j=i+1, i+2, \ldots n ; k=1,2, \ldots, \# L_{i j}^{k}-1 \\
\sigma \leq \tilde{x}_{i j}^{k} \leq 1 \text { with } \sum_{k=1}^{\# L_{i j}} \tilde{x}_{i j}^{k}=1, \quad i=1,2, \ldots, n-1 ; j=i+1, i+2, \ldots n \\
\left.\tilde{\varepsilon}_{i j}^{k+}, \tilde{\varepsilon}_{i j}^{k-} \geq 0 \text { with } \tilde{\varepsilon}_{i j}^{k+} \tilde{\varepsilon}_{i j}^{k-}=0, \quad i=1,2, \ldots, n-1 ; j=i+1, i+2, \ldots n ; k=1,2, \ldots, \# L_{i j}^{k}\right) \\
\tilde{\varphi}_{i j}^{+}, \tilde{\varphi}_{i j}^{k} \geq 0 \text { with } \tilde{\varphi}_{i j}^{+} \tilde{\varphi}_{i j}^{-}=0, \quad i=1,2, \ldots, n-1 ; j=i+1, i+2, \ldots n \\
\delta_{i j h}^{+}, \delta_{i j h}^{-} \geq 0 \text { with } \delta_{i j h}^{+} \delta_{i j h}^{-}=0, \quad 1 \leq i<j<h \leq n .
\end{array}\right.
\end{aligned}
$$

Clearly, model (21) has feasible solutions, e.g., the matrix $\bar{R}=\left(\bar{L}(x)_{i j}\right)_{n \times n}$, where each $\bar{L}(x)_{i j}=\left\{s_{\tau}(1)\right\}$ is a feasible solution. According to the Weierstrass theorem [1] and the fact that the objective function is bounded, model (21) has at least one optimal solution.

\section{Stage 2 Optimization}

For the situation where there is at least one pair of the solutions for model (21), theoretically, any optimal solution can be used as a final feasible solution. However, in practical decision-making problems, decision-makers usually wish to select the final solution for deciding among multiple optimal solutions 
based on individual wishes and preferences.

In the process of consistency improvement, we tend to want the number of elements to be adjusted as few as possible, so that decision-makers are more likely to be satisfied with the adjustment results. Therefore, to consider the satisfaction of decision-makers, we construct the second-stage optimization model based on the minimum number of adjusted elements to find the final solution. There are two information dimensions in the CPLTS, and its uncertainty is mainly caused by the probability dimension. Therefore, in the process of consistency adjustment, the adjustment of the probability is first task that we perform. However, in some cases, adjusting only the probability may not satisfy the requirements. For decision-makers, it is better to have fewer changes in the original LT information. Thus, a 0-1 optimization model (22) can be constructed, as follows:

$$
\begin{aligned}
& \min M=\sum_{i=1}^{n-1} \sum_{j=i+1}^{n} \sum_{k=1}^{\# L_{i j}} \psi_{i j}^{k} \\
& \text { s.t. }\left\{\begin{array}{l}
\frac{1}{2 \tau n(n-1)} \sum_{i=1}^{n-1} \sum_{j=i+1}^{n} \sum_{k=1}^{\# L_{i j}} \frac{1}{\# L_{i j}}\left(\tilde{\varepsilon}_{i j}^{k+}+\tilde{\varepsilon}_{i j}^{k-}\right)+\frac{1}{2 \tau n(n-1)} \sum_{i=1}^{n-1} \sum_{j=i+1}^{n}\left(\tilde{\varphi}_{i j}^{+}+\tilde{\varphi}_{i j}^{-}\right)=d_{*} \\
\psi_{i j}^{k+} \in\left\{\tilde{\varepsilon}_{i j}^{k-}\right)\left(1-\psi_{i j}^{k}\right)=0, i=1,2, \ldots, n-1 ; j=i+1, i+2, \ldots n ; k=1,2, \ldots, \# L_{i j} \\
\text { All other constraints in model }(18) .
\end{array}\right.
\end{aligned}
$$

\section{Stage 3 Optimization}

As model (22) has at least one optimal solution, the goal of the third stage is to minimize the uncertainty of the CPLPR. For the adjusted CPLPR $\tilde{R}=\left(\tilde{L}(x)_{i j}\right)_{n \times n}$, the total uncertainty of the information is given as follows:

$$
U=-\sum_{i=1}^{n-1} \sum_{j=i+1}^{n} \sum_{k=1}^{\# \tilde{L}_{i j}} x_{i j}^{k} \log _{2} x_{i j}^{k}
$$

Let $M_{*}$ be the optimal objective value for model (22). Then, the third-stage optimization model can be expressed as follows:

$$
\begin{aligned}
& \min U=-\sum_{i=1}^{n-1} \sum_{j=i+1}^{n} \sum_{k=1}^{\# \tilde{L}_{i j}} x_{i j}^{k} \log _{2} x_{i j}^{k} \\
& \text { s.t. }\left\{\begin{array}{l}
\sum_{i=1}^{n-1} \sum_{j=i+1}^{n} \sum_{k=1}^{\# L_{i j}} \psi_{i j}^{k}=M_{*} \\
\text { All other constraints in model (19). }
\end{array}\right.
\end{aligned}
$$

By solving model (24), the adjusted $\tilde{r}_{i j}^{k}$ and $\tilde{x}_{i j}^{k}$ can be obtained. Thus, a CPLPR $\tilde{R}=\left(\tilde{L}(x)_{i j}\right)_{n \times n}$ with acceptable consistency can be obtained, as follows:

$$
\tilde{L}(x)_{i j}= \begin{cases}\left\{s_{\tilde{r}_{i j}^{k}}\left(\tilde{x}_{i j}^{k}\right)\right\} & \text { if } i<j ; k=1,2, \ldots, \# L(x)_{i j} \\ \left\{s_{\tau}\right\} & \text { if } i=j \\ \left\{s_{2 \tau-\tilde{r}_{i j}^{k}}\left(\tilde{x}_{i j}^{k}\right)\right\} & \text { if } i>j ; k=1,2, \ldots, \# L(x)_{i j} .\end{cases}
$$

Remark 6. In complex decision-making problems, a decision-making optimization model with at least one optimal solution is not uncommon. It is an interesting topic for decision-makers to select the final solution according to the actual decision-making situation or personal preference. For example, some mixed integer programming models, or the "core" concept used in the dominance based rough set approach [10] are worthy of in-depth study. Additionally, according to the actual requirements of decision-making or the need of solving the model, the decision-maker can substitute the objective function in the model (24) with formula (14).

Remark 7. The multi-stage models proposed in this paper are lexicographic in nature, and it is obvious 
that there are other types of orders. Although a different order may lead to different final recommendation results, the central idea of the proposed framework is applicable. Instead of using sequential optimization, another method involves treating the sequential objective as a set of multiple objectives of a complex optimization, which is also known as multi-objective optimization. In this method, the multiple objectives necessitate a tradeoff analysis, which is also an interesting research topic for the future.

\subsection{Derivation of priority weight vector from InPLPR}

Gao et al. [9] reported that if a PLPR $P$ is multiplicatively consistent, it can be represented by the following formula:

$$
\frac{1}{2 \tau} E_{i j}=\frac{w_{i}}{w_{i}+w_{j}}=\frac{1}{2 \tau} \sum_{k=1}^{\# L(p)} r_{i j}^{k} p_{i j}^{k},
$$

where $W=\left(w_{1}, w_{2}, \ldots, w_{n}\right)^{T}$ represents the priority weight vector $P$ with $w_{i} \geq 0$ and $\sum_{i=1}^{n} w_{i}=1$.

Inspired by this idea, we have the following theorem.

Theorem 1. For an InPLPR $R=\left(L(x)_{i j}\right)_{n \times n}$ whose corresponding CPLPR is $\bar{R}=\left(\bar{L}(x)_{i j}\right)_{n \times n}, \bar{E}_{i j}$ represents the expected value of the $\bar{L}(x)_{i j}$. Then, $R$ is expected to be a multiplicatively consistent InPLPR if and only if there exists a positive normalized priority weight vector $W=\left(w_{1}, w_{2}, \ldots, w_{n}\right)^{T}$ with $w_{i} \geq 0$ and $\sum_{i=1}^{n} w_{i}=1$, such that for all $i$ and $j(i, j=1,2, \ldots, n)$,

$$
\frac{w_{i}}{w_{j}}=\frac{\bar{E}_{i j}}{\bar{E}_{j i}}=\frac{\sum_{k=1}^{\# \bar{L}(x)_{i j}} r_{i j}^{k} x_{i j}^{k}}{\sum_{k=1}^{\# \bar{L}(x)_{j i}} r_{j i}^{k} x_{j i}^{k}},
$$

where $\bar{L}(x)_{i j}=\left\{\bar{L}_{i j}^{k}\left(x_{i j}^{k}\right) \mid k=1,2, \ldots, \# \bar{L}(x)_{i j}\right\}$ represents an element of CPLPR $\bar{R}, \quad r_{i j}^{k}$ represents the subscript of $\bar{L}_{i j}^{k}$, and $x_{i j}^{k}$ represents the occurrence probability of $\bar{L}_{i j}^{k}$.

Proof. Because $\bar{R}=\left(\bar{L}(x)_{i j}\right)_{n \times n}$ is a CPLPR, it satisfies Eq. (26). The above equation (27) can be obtained through transformation.

Formula (27) is only true if the InPLPR is a preference relation with completely expected multiplicative consistency. However, for various reasons, it is difficult to obtain the completely consistent preference relation. Therefore, there are deviations on both sides of the equals sign of formula (27). A smaller deviation corresponds to a more consistent and reasonable preference relation. Therefore, to derive a reasonable priority weight vector, the following model is established for minimizing the deviation:

$$
\begin{aligned}
& \min \operatorname{Dev}=\sum_{i=1}^{n} \sum_{j=1}^{n}\left(w_{i} \sum_{k=1}^{\# \bar{L}(x) j_{j i}} r_{j i}^{k} x_{j i}^{k}-w_{j} \sum_{k=1}^{\# \bar{L}(x)_{i j}} r_{i j}^{k} x_{i j}^{k}\right)^{2} . \\
& \text { s.t. } \sum_{i=1}^{n} w_{i}=1, \quad 0 \leq w_{i} \leq 1 .
\end{aligned}
$$

To facilitate the solution of the model, we can apply a logarithmic transformation to Eq. (27), which yields the following equation:

$$
\ln \frac{w_{i}}{w_{j}}=\ln \frac{\sum_{k=1}^{\# \bar{L}(x)_{i j}} r_{i j}^{k} x_{i j}^{k}}{\sum_{k=1}^{\# \bar{L}(x)_{j i}} r_{j i}^{k} x_{j i}^{k}} .
$$


Theorem 2. Suppose that $R=\left(L(x)_{i j}\right)_{n \times n}$ is an InPLPR and $\bar{R}=\left(\bar{L}(x)_{i j}\right)_{n \times n}$ is its corresponding CPLPR, and suppose that $W=\left(w_{1}, w_{2}, \ldots, w_{n}\right)^{T}$ is the normalized priority weight vector. Then, according to the expected multiplicative consistency of the InPLPR, the weight vector $W$ can be derived by the following programming model:

$$
\begin{aligned}
& \min D=\sum_{i=1}^{n} \sum_{j=1}^{n}\left(\ln w_{i}+\ln \sum_{k=1}^{\# \bar{L}(x)_{j i}} r_{j i}^{k} x_{j i}^{k}-\ln w_{j}-\ln \sum_{k=1}^{\# \bar{L}(x)_{i j}} r_{i j}^{k} x_{i j}^{k}\right)^{2} . \\
& \text { s.t. } \sum_{i=1}^{n} w_{i}=1, \quad 0 \leq w_{i} \leq 1 .
\end{aligned}
$$

Then, by solving this model mathematically, we obtain

$$
w_{i}=\frac{\sqrt[n]{\prod_{j=1}^{n} \frac{\sum_{k=1}^{\# \bar{L}(x)_{i j}} r_{i j}^{k} x_{i j}^{k}}{\sum_{k=1}^{\# \bar{L}(x)_{j i}} r_{j i}^{k} x_{j i}^{k}}}}{\sum_{s=1}^{n} \sqrt[n]{\prod_{j=1}^{n} \frac{\sum_{k=1}^{\# \bar{L}(x)_{s j}} r_{s j}^{k} x_{s j}^{k}}{\sum_{k=1}^{\# \bar{L}(x)_{j s}} r_{j s}^{k} x_{j s}^{k}}}} i, j, s=1,2, \ldots, n .
$$

Proof. Using the Lagrange method, model (31) can be converted into the following unconstrained function:

$$
L(w, \lambda)=\sum_{i=1}^{n} \sum_{j=1}^{n}\left(\ln w_{i}+\ln \sum_{k=1}^{\# \bar{L}(x)_{j i}} r_{j i}^{k} x_{j i}^{k}-\ln w_{j}-\ln \sum_{k=1}^{\# \bar{L}(x)_{i j}} r_{i j}^{k} x_{i j}^{k}\right)^{2}+4 \lambda\left(\sum_{i=1}^{n} w_{i}-1\right) .
$$

Clearly, $\frac{\partial L(w, \lambda)}{\partial \lambda}=\sum_{i=1}^{n} w_{i}-1=0$. By letting $\frac{\partial L(w, \lambda)}{\partial w_{i}}=0$, we obtain

$$
4 \sum_{j=1}^{n} \frac{\left(\ln w_{i}+\ln \sum_{k=1}^{\# \bar{L}(x)_{j i}} r_{j i}^{k} x_{j i}^{k}-\ln w_{j}-\ln \sum_{k=1}^{\# \bar{L}(x)_{i j}} r_{i j}^{k} x_{i j}^{k}\right)}{w_{i}}+4 \lambda=0 .
$$

Accordingly, $\sum_{j=1}^{n}\left(\ln w_{i}+\ln \sum_{k=1}^{\# \bar{L}(x)_{j i}} r_{j i}^{k} x_{j i}^{k}-\ln w_{j}-\ln \sum_{k=1}^{\# \bar{L}(x)_{i j}} r_{i j}^{k} x_{i j}^{k}\right)+\lambda w_{i}=0$.

Thus, $\sum_{i=1}^{n}\left[\sum_{j=1}^{n}\left(\ln w_{i}+\ln \sum_{k=1}^{\# \bar{L}(x)_{j i}} r_{j i}^{k} x_{j i}^{k}-\ln w_{j}-\ln \sum_{k=1}^{\# \bar{L}(x)_{i j}} r_{i j}^{k} x_{i j}^{k}\right)+\lambda w_{i}\right]=0$.

Because $\quad \frac{\sum_{k=1}^{\# \bar{L}(x)_{i j}} r_{i j}^{k} x_{i j}^{k}}{\sum_{k=1}^{\# \bar{L}(x)_{j i}} r_{j i}^{k} x_{j i}^{k}} \frac{\sum_{k=1}^{\# \bar{L}(x)_{j i}} r_{j i}^{k} x_{j i}^{k}}{\sum_{k=1}^{\# \bar{L}(x)_{i j}} r_{i j}^{k} x_{i j}^{k}}=1 \quad, \quad \prod_{i}^{n} \prod_{j=1}^{n} \frac{\sum_{k=1}^{\# \bar{L}(x)_{i j}} r_{i j}^{k} x_{i j}^{k}}{\sum_{k=1}^{\# \bar{L}(x)_{j i}} r_{j i}^{k} x_{j i}^{k}}=1 \quad ; \quad$ therefore, $\sum_{i=1}^{n} \sum_{j=1}^{n}\left(\ln \sum_{k=1}^{\# \bar{L}(x)_{i j}} r_{i j}^{k} x_{i j}^{k}-\ln \sum_{k=1}^{\# \bar{L}(x)_{j i}} r_{j i}^{k} x_{j i}^{k}\right)=\sum_{i=1}^{n} \sum_{j=1}^{n}\left(\ln \frac{\sum_{k=1}^{\# \bar{L}(x)_{i j}} r_{i j}^{k} x_{i j}^{k}}{\sum_{k=1}^{\# \bar{L}(x)_{j i}} r_{j i}^{k} x_{j i}^{k}}\right)=\ln \prod_{i}^{n} \prod_{j=1}^{n} \frac{\sum_{k=1}^{\# \bar{L}(x)_{i j}} r_{i j}^{k} x_{i j}^{k}}{\sum_{k=1}^{\# \bar{L}(x)_{j i}} r_{j i}^{k} x_{j i}^{k}}=0$. Because $\sum_{i=1}^{n} \sum_{j=1}^{n}\left(\ln w_{i}-\ln w_{j}\right)=0, \sum_{i=1}^{n} \sum_{j=1}^{n}\left(\log _{\beta} \sum_{k=1}^{\# \bar{L}(x)_{i j}} r_{i j}^{k} x_{i j}^{k}-\log _{\beta} \sum_{k=1}^{\# \bar{L}(x)_{j i}} r_{j i}^{k} x_{j i}^{k}\right)=0$; then, $\sum_{i=1}^{n} \sum_{j=1}^{n} \lambda w_{i}=0$. Thus, $\sum_{i=1}^{n} \sum_{j=1}^{n} \lambda w_{i}=n \lambda \sum_{i=1}^{n} w_{i}=n \lambda=0$, and $\lambda=0$. Hence, we can obtain the following equations:

$$
\left\{\begin{array}{l}
\sum_{j=1}^{n}\left(\ln w_{i}+\ln \sum_{k=1}^{\# \bar{L}(x)_{j i}} r_{j i}^{k} x_{j i}^{k}-\ln w_{j}-\ln \sum_{k=1}^{\# \bar{L}(x)_{i j}} r_{i j}^{k} x_{i j}^{k}\right)=0 \\
\sum_{i=1}^{n} w_{i}=1, \quad 0 \leq w_{i} \leq 1 .
\end{array}\right.
$$

It is easy to verify that $w_{i}$ of the form 
satisfies the above equations. The proof of Theorem 2 is completed.

\subsection{Decision-making algorithm considering consistency of InPLPR}

Considering the foregoing analysis, we propose a decision-making algorithm based on multi-stage consistency optimization model for PDM with an InPLPR.

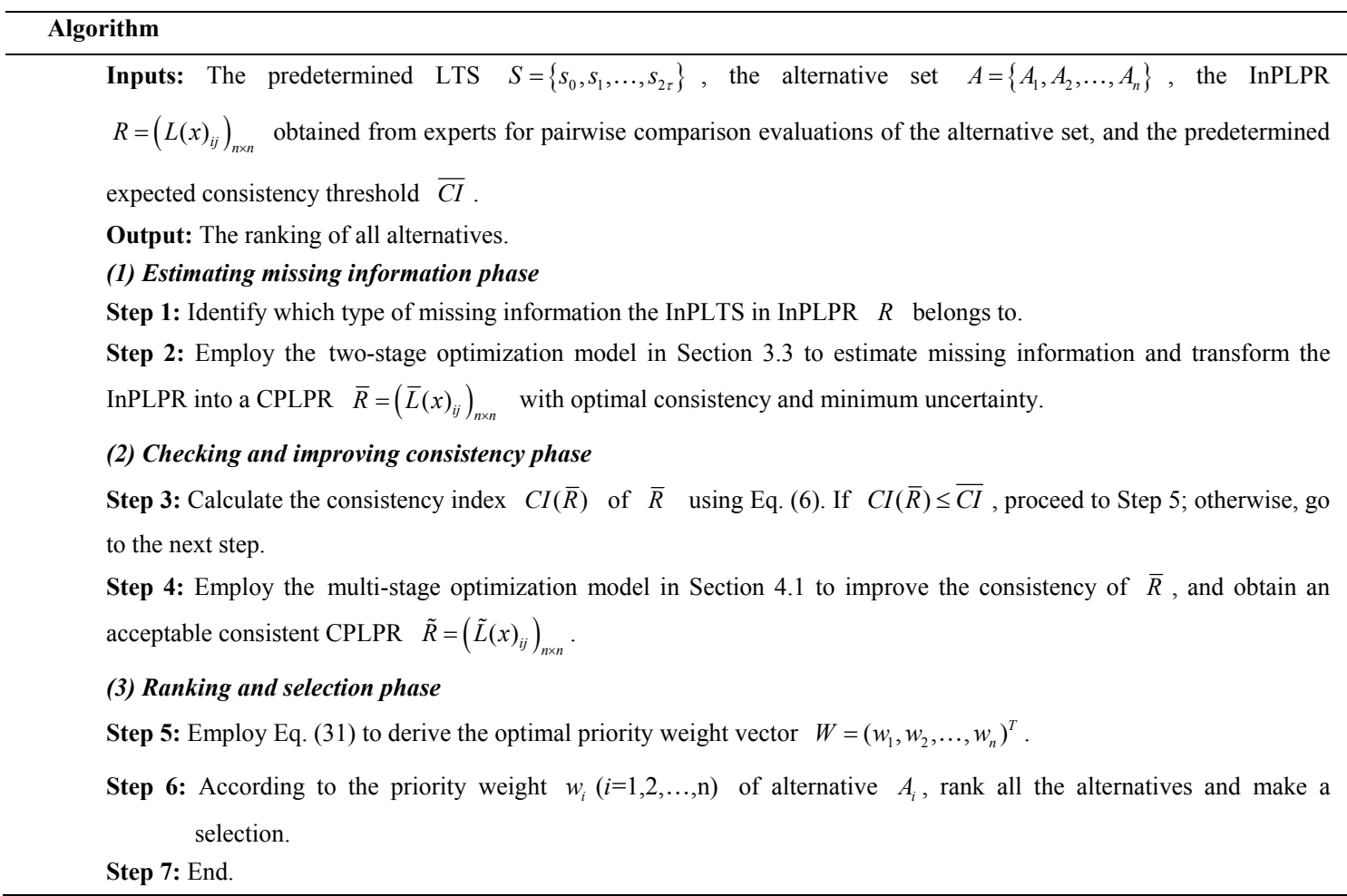

The procedure of the introduced algorithm is depicted in Fig. 1.

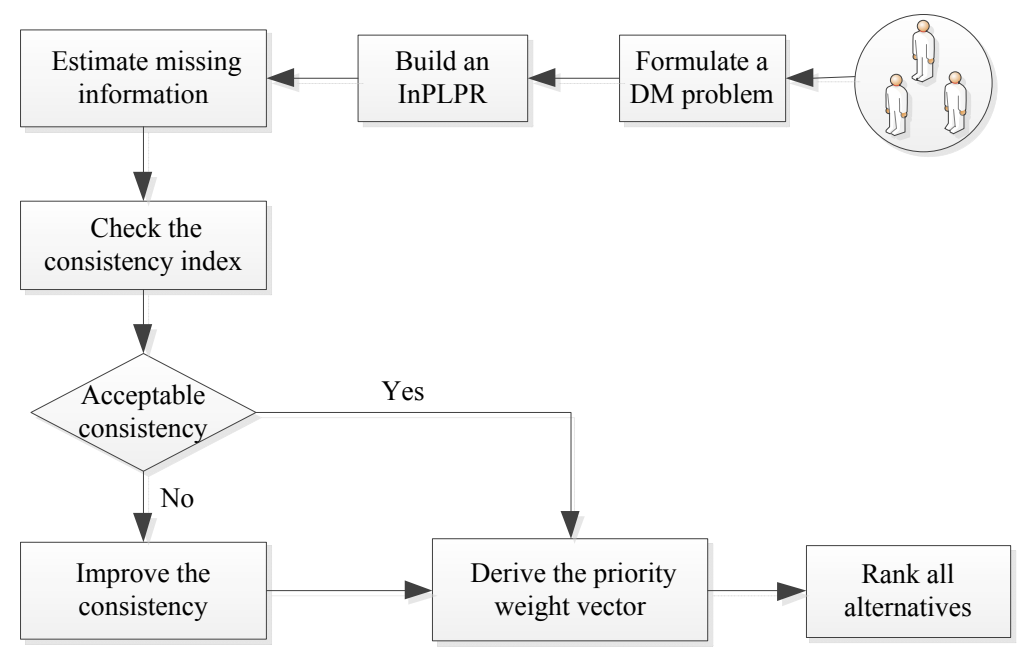

Fig. 1. Procedure of the proposed algorithm 


\section{Numerical case illustration and comparative analyses}

\subsection{Numerical case}

In this subsection, a practical case is presented to illustrate the practicality of the proposed decision-making algorithm.

To more effectively cultivate research-based graduate students, $S$ university in China has begun to implement a successive master-doctor project. The objective is to select a group of first-year graduate students with scientific research potential to pursue a doctoral degree for at least four years. This project is beneficial to both university and students. However, it has quota restrictions, requiring doctoral supervisors with high degrees of academic achievement to select at most one student among the postgraduates under their supervision. There is a doctoral supervisor with four qualified graduate students. He must select one of the best students to participate in this project. Because of time constraints, he calls his research team together and consults other teachers and uses an InPLPR to evaluate the performance of these graduate students via pairwise comparisons. Then, the proposed algorithm is employed to check and improve the consistency of the InPLPR provided, and a selection process is conducted to select the best candidate.

The four candidates are represented by $A_{1}, A_{2}, A_{3}$, and $A_{4}$, respectively, and the InPLPR matrix provided is $R=\left(L(x)_{i j}\right)_{4 \times 4}$ according to a pairwise comparison between four graduate students. Here, we use the LTS: $S=\left\{s_{0}=\right.$ particularly bad, $s_{1}=$ very bad, $s_{2}=$ bad, $s_{3}=$ somewhat bad, $s_{4}=$ medium, $s_{5}=$ somewhat good, $s_{6}=$ good, $s_{7}=$ very good, $s_{8}=$ particularly good $\}$. The evaluation matrix is shown below.

$$
R=\left[\begin{array}{lccc}
\left\{s_{4}(1)\right\} & \left\{s_{2}(1)\right\} & \left\{s_{1}\left(x_{13,1}\right), s_{3}\left(x_{13,2}\right), s_{6}\left(x_{13,3}\right)\right\} & \left\{s_{1}(1)\right\} \\
\left\{s_{6}(1)\right\} & \left\{s_{4}(1)\right\} & \left.s_{2}\left(x_{23,1}\right), s_{6}\left(x_{23,2}\right)\right\} & \left\{s_{3}(1)\right\} \\
\left\{s_{7}\left(x_{31,1}\right), s_{5}\left(x_{31,2}\right), s_{2}\left(x_{31,3}\right)\right\} & \left\{s_{6}\left(x_{32,1}\right), s_{2}\left(x_{32,2}\right)\right\} & \left\{s_{4}(1)\right\} & \left\{s_{2}(0.7), s_{3}(0.2)\right\} \\
\left\{s_{7}(1)\right\} & \left\{s_{5}(1)\right\} & \left\{s_{6}(0.7), s_{5}(0.2)\right\} & \left\{s_{4}(1)\right\}
\end{array}\right]
$$

Using the proposed multi-stage optimization algorithm, we select the optimal candidate according to the foregoing preference evaluation information. The specific steps are as follows:

Step 1: By analyzing the upper triangular matrix, we find that $R(x)_{13}$ and $R(x)_{23}$ are identified as MP-InPLTSs, and $R(x)_{34}$ is identified as an MLT-InPLTS.

Step 2: The two-stage models presented in Section 3.3 are employed to estimate the missing information of $R$. First, $R$ is substituted into model (9), and the model is solved via LINGO 11.0. Then, we have a CPLPR $\bar{R}_{\text {Stage }_{1}}$ based on the optimal solution. To seek the solution conveniently and reasonably, we set $\sigma=0.01$ in this study.

$\bar{R}_{\text {Stage }_{1}}=\left[\begin{array}{llll}\left\{s_{4}(1)\right\} & \left\{s_{2}(1)\right\} & \left\{s_{1}(\mathbf{0 . 0 9}), s_{3}(\mathbf{0 . 9}), s_{6}(\mathbf{0 . 0 1})\right\}\left\{s_{1}(1)\right\} \\ \left\{s_{6}(1)\right\} & \left\{s_{4}(1)\right\} & \left\{s_{2}(\mathbf{0 . 2 5 2}), s_{6}(\mathbf{0 . 7 4 8})\right\} & \left\{s_{3}(1)\right\} \\ \left\{s_{7}(\mathbf{0 . 0 9}), s_{5}(\mathbf{( 0 . 9}), s_{2}(\mathbf{0 . 0 1})\right\}\left\{s_{6}(\mathbf{0 . 2 5 2}), s_{2}(\mathbf{0 . 7 4 8})\right\} & \left\{s_{4}(1)\right\} & \left\{\boldsymbol{s}_{1.23}(\mathbf{0 . 1}), s_{2}(0.7), s_{3}(0.2)\right\} \\ \left\{s_{7}(1)\right\} & \left\{s_{4}(1)\right\} & \left\{\mathbf{s}_{\mathbf{6 . 7 7}}(\mathbf{0 . 1}), s_{6}(0.7), s_{5}(0.2)\right\} & \left\{s_{4}(1)\right\}\end{array}\right]$

However, as it is unclear whether the uncertainty of CPLPR $\bar{R}_{\text {Stage }_{1}}$ is minimal, the second-stage model (12) is implemented, and the following CPLPR $\bar{R}_{\text {Stage }_{1}}$ with minimum uncertainty is obtained: 
Step 3: Eq. (6) is used to calculate the consistency index $C I$ of $\bar{R}_{\text {Stage }_{2}}$. We obtain $C I\left(\bar{R}_{\text {tage }_{2}}\right)=0.168 \geq \overline{C I}=0.1$, which indicates that $\bar{R}_{\text {Stage }_{2}}$ is a matrix with unacceptable consistency. Thus, we proceed to the next step.

Step 4: The multi-stage optimization model in Section 4.1 is used to improve the consistency of $\bar{R}_{\text {Stage }_{2}}$. First, $\bar{R}_{\text {Stage }_{2}}$ is substituted into model (21), and the model is solved using LINGO 11.0. Then, we have a CPLPR $\tilde{R}_{\text {Stage }_{1}}$ with minimum information distortion based on the optimal solution.

$\tilde{R}_{\text {Stage }}=\left[\begin{array}{lccc}\left\{s_{4}(1)\right\} & \left\{s_{2}(1)\right\} & \left\{s_{1}(\mathbf{0 . 0 4 9}), s_{3}(\mathbf{0 . 9 1 9}), s_{6}(\mathbf{0 . 0 3 2})\right\} & \left\{\boldsymbol{s}_{1.126}(1)\right\} \\ \left\{s_{6}(1)\right\} & \left\{s_{4}(1)\right\} & \left\{s_{2}(0.214), s_{6}(0.786)\right\} & \left\{s_{3}(1)\right\} \\ \left\{s_{7}(\mathbf{0 . 0 4 9}), s_{5}(\mathbf{0 . 9 1 9}), s_{2}(\mathbf{0 . 0 3 2})\right\} & \left.\left\{s_{6}(0.214), s_{2}(0.786)\right\} s_{4}(1)\right\} & \left\{s_{0}(\mathbf{0 . 0 4 3}), s_{2}(\mathbf{0 . 8 7 1}), s_{3}(\mathbf{0 . 0 8 6})\right\} \\ \left\{\boldsymbol{s}_{\mathbf{6 . 8 7 4}}(1)\right\} & \left\{s_{4}(1)\right\} & \left\{s_{8}(\mathbf{0 . 0 4 3}), s_{6}(\mathbf{0 . 8 7 1}), s_{5}(\mathbf{( 0 . 0 8 6})\right\}\left\{s_{4}(1)\right\}\end{array}\right]$

Next, the second-stage model (22) is implemented, and the following CPLPR $\tilde{R}_{\text {Stage }_{2}}$ with the minimum number of adjusted LTs is obtained.

$\tilde{R}_{\text {Stage }_{2}}=\left[\begin{array}{llll}\left\{s_{4}(1)\right\} & \left\{s_{2}(1)\right\} & \left\{s_{1}(0.049), s_{3}(0.919), s_{6}(0.032)\right\} & \left\{\mathbf{s}_{1.126}(1)\right\} \\ \left\{s_{6}(1)\right\} & \left\{s_{4}(1)\right\} & \left\{s_{2}(0.214), s_{6}(0.786)\right\} & \left\{s_{3}(1)\right\} \\ \left\{s_{7}(0.049), s_{5}(0.919), s_{2}(0.032)\right\}\left\{s_{6}(0.214), s_{2}(0.786)\right\} & \left\{s_{4}(1)\right\} & \left\{s_{0}(0.043), s_{2}(0.871), s_{3}(0.086)\right\} \\ \left\{\mathbf{s}_{6.874}(1)\right\} & \left\{s_{4}(1)\right\} & \left\{s_{8}(0.043), s_{6}(0.871), s_{5}(0.086)\right\} & \left\{s_{4}(1)\right\}\end{array}\right]$

Clearly, the minimum adjustment number of LTs is 1 when we only analyze the upper triangular matrix. According to this premise, we implement the third-stage model (24). Final, we obtain the CPLPR $\tilde{R}_{\text {Stage }_{3}}=\left(\tilde{L}(x)_{i j}\right)_{4 \times 4}$ with minimum information uncertainty.

$\tilde{R}_{\text {Stage }_{3}}=\left[\begin{array}{lccl}\left\{s_{4}(1)\right\} & \left\{s_{2}(1)\right\} & \left\{s_{1}(0.015), s_{3}(0.975), s_{6}(0.01)\right\}\left\{\boldsymbol{s}_{\mathbf{1 . 1 2 6}}(1)\right\} \\ \left\{s_{6}(1)\right\} & \left\{s_{4}(1)\right\} & \left\{s_{2}(0.214), s_{6}(0.786)\right\} & \left\{s_{3}(1)\right\} \\ \left\{s_{7}(0.015), s_{5}(0.975), s_{2}(0.01)\right\}\left\{s_{6}(0.214), s_{2}(0.786)\right\}\left\{s_{4}(1)\right\} & \left\{s_{0}(\mathbf{0 . 0 1}), s_{2}(\mathbf{0 . 9 7}), s_{3}(\mathbf{0 . 0 2})\right\} \\ \left\{\boldsymbol{s}_{\mathbf{6 . 8 7 4}}(1)\right\} & \left\{s_{4}(1)\right\} & \left\{s_{8}(\mathbf{0 . 0 1}), s_{6}(\mathbf{0 . 9 7}), s_{5}(\mathbf{0 . 0 2})\right\} & \left\{s_{4}(1)\right\}\end{array}\right]$

Here, the consistency index is $C I\left(\tilde{R}_{\text {tage }_{3}}\right)=0.1=\overline{C I}$. Intuitively, only the LT of $\tilde{L}(x)_{14}$ and the probability distribution of $\tilde{L}(x)_{34}$ are adjusted. The deviation degree between $\bar{R}_{\text {Stage }_{2}}$ and $\tilde{R}_{\text {Stage }_{3}}$ is obtained as $d\left(\bar{R}_{\text {Stage }_{2}}, \tilde{R}_{\text {Stage }_{3}}\right)=0.003$. Clearly, the CPLPR $\tilde{R}_{\text {Stage }_{3}}$ with acceptable consistency is very close to the initial CPLPR $\bar{R}_{\text {Stage }_{2}}$, indicating that $\tilde{R}_{\text {Stage }_{3}}$ causes very little information distortion to $\bar{R}_{\text {Stage }_{2}}$ and preserves the original preference information well.

Step 5: Eq. (31) is used to derive the optimal normalized priority weight vector, as follows:

$$
w_{1}=0.087, w_{2}=0.276, w_{3}=0.153, w_{4}=0.483 \text {. }
$$

Step 6: With these priority weights, the ranking of all the alternatives is $A_{4} \succ A_{2} \succ A_{3} \succ A_{1}$. Thus, $A_{4}$ is the best graduate student.

Step 7: End.

\subsection{Validity of proposed algorithm}

In solving decision-making problems, the rank reversal phenomenon is often inevitable. Wang and Triantaphyllou [34] pointed out that the rank reversal phenomenon occurs in not only the AHP method 
but also other decision-making methods. Wang and Luo [35] proposed three criteria for evaluating the

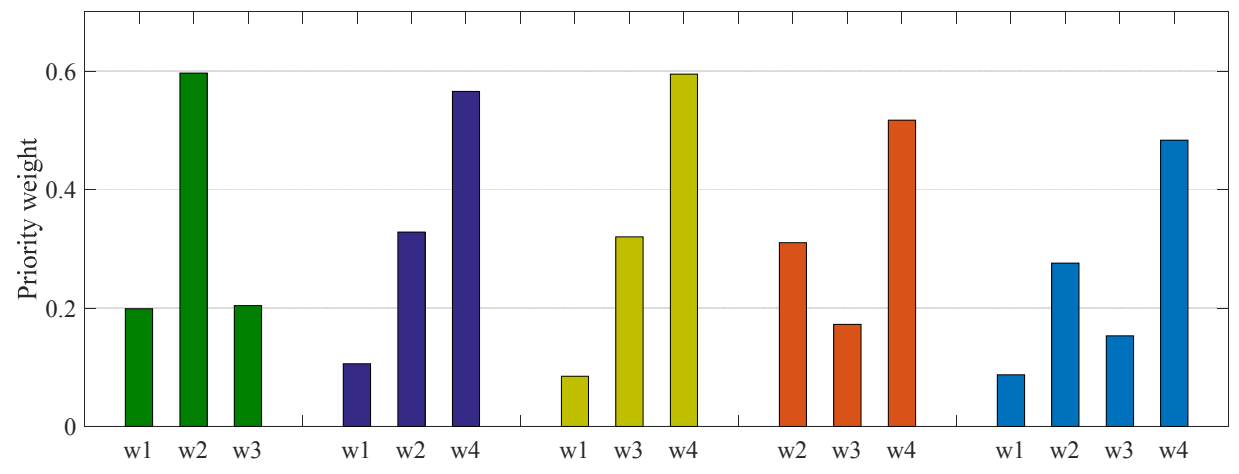

Fig. 2 Priority weights of alternatives from different scenarios

As shown in Fig. 2, when we extract alternative subset $\left\{A_{1}, A_{2}, A_{3}\right\}$, the ranking result is $A_{2} \succ A_{3} \succ A_{1}$, which is consistent with the original ranking result $A_{4} \succ A_{2} \succ A_{3} \succ A_{1}$. Similarly, when we extract alternative subsets $\left\{A_{1}, A_{2}, A_{4}\right\},\left\{A_{1}, A_{3}, A_{4}\right\}$, and $\left\{A_{2}, A_{3}, A_{4}\right\}$, we obtain the ranking results $A_{4} \succ A_{2} \succ A_{1}, A_{4} \succ A_{3} \succ A_{1}$, and $A_{4} \succ A_{2} \succ A_{3}$, which are also in accordance with the original ranking result. These results are in complete agreement with Criterion I; thus, the proposed algorithm is effective under Criterion I and can effectively avoid the rank reversal phenomenon.

(2) Validity test for proposed algorithm using Test Criteria II and III

The validity test for Criteria II and III can be analyzed together. The original example with alternative set $\left\{A_{1}, A_{2}, A_{3}, A_{4}\right\}$ is decomposed into two sub-problems with alternative sets $\left\{A_{1}, A_{2}, A_{3}\right\}$ and $\left\{A_{1}, A_{2}, A_{4}\right\}$. Using the proposed algorithm, we obtain the ranking results of the two sub-problems 
$A_{2} \succ A_{3} \succ A_{1}$ and $A_{4} \succ A_{2} \succ A_{1}$. These two ranking results are combined using transitivity, and the overall ranking results of the un-decomposed problem are obtained as $A_{4} \succ A_{2} \succ A_{3} \succ A_{1}$, which is consistent with the original ranking result. Therefore, the proposed algorithm is also effective under

\section{Criteria II and III.}

\subsection{Comparative analyses and discussion}

To verify the comprehensiveness and superiority of the proposed algorithm, we performed two comparisons: a comparison with typical decision-making methods considering the consistency of PLPRs and a comparison with methods considering the possibility distribution generation for HFLPRs. The mean deviation $\theta=d(\bar{R}, \tilde{R})$ based on Eq. (19), i.e., the deviation between the adjusted matrix and the original matrix, was used to compare the information distortion of the algorithms.

\subsubsection{Comparative analyses with method considering consistency of PLPRs}

\section{(1) Comparative analyses with Zhang et al.'s method [47]}

In this subsection, we compare the proposed algorithm with the method of [47] considering the ordinal consistency of PLPRs. It should be noted that the LTS used in [47] is the subscript-symmetric linguistic evaluation scale $\left\{s_{-3}, s_{-2}, s_{-1}, s_{0}, s_{1}, s_{2}, s_{3}\right\}$. Therefore, before the experiment, we must convert the data into the LTS of $\left\{s_{0}, s_{1}, \ldots, s_{6}\right\}$; e.g., $s_{-1}$ can be converted into $s_{2}$. These operations do not affect the experimental results. Additionally, for the PLTS used in [47], when the sum of the probabilities of all the LTs is $<1$, it is called the MLT-InPLTS in this paper. Zhang et al.'s method [47] adopts the normalization method to deal with this case and obtain the normalized PLPR, which is called the CPLPR in this study.

To compare the proposed algorithm with Zhang et al.'s method [47], we performed the following operations.

Operation 1: Employ the Zhang et al.'s method [47] to handle the original PLPR $R^{\text {original }}$ in [47], and obtain the CPLPR $\bar{R}_{1}$ and a final acceptable consistent matrix $\tilde{R}_{1}$. Then, rank the alternatives. Operation 2: Employ the proposed algorithm to handle the original PLPR $R^{\text {original }}$. Obtain the CPLPR $\bar{R}_{2}$ and an acceptable consistent matrix $\tilde{R}_{2}$, and then rank the alternatives. Operation 3: Employ the proposed ranking method to rank the alternatives according to the matrix $\tilde{R}_{1}$.

Because Zhang et al.'s method [47] adopts the ordinal consistency, it cannot reflect the level of consistency from specific data. Thus, in this experiment, we employ the proposed consistency index $C I$ to measure the consistency level of $\tilde{R}_{1}$ and $\tilde{R}_{2}$. Additionally, to perform a comprehensive comparison, the mean deviation $\theta$, the number of adjusted LTs, and the number of adjusted rounds are calculated or counted. The comparison results are presented in Table 1 . The matrices $R^{\text {original }}, \bar{R}_{1}, \tilde{R}_{1}, \bar{R}_{2}$, and $\tilde{R}_{2}$ are presented in Appendix I.

Table 1. Experimental results of the two methods

\begin{tabular}{ccccccc}
\hline Methods & $C I\left(\bar{R}_{i}\right)(i=1,2)$ & $C I\left(\tilde{R}_{i}\right)(i=1,2)$ & Ranking & $\theta$ & Adjusted LTs & Adjusted round \\
\hline $\begin{array}{c}\text { Zhang et al.'s } \\
\text { method [47] }\end{array}$ & 1.706 & 1.660 & $x_{1} \succ x_{4} \succ x_{2} \succ x_{5} \succ x_{3}$ & 0.061 & 2 & 2 \\
$\begin{array}{c}\text { Proposed } \\
\text { algorithm }\end{array}$ & 0.942 & 0.1 & $x_{1} \succ x_{2} \succ x_{4} \succ x_{3} \succ x_{5}$ & 0.024 & 1 & 1 \\
\hline
\end{tabular}

Note: The ranking obtained by Operation 3 is $x_{1} \succ x_{4} \succ x_{2} \succ x_{5} \succ x_{3}$.

As indicated by Table 1, the consistency of the estimated CPLPR obtained by the proposed algorithm is better than that obtained using Zhang et al.'s method; that is, the proposed information completion 
mechanism is more accurate. For improving the consistency, the proposed method uses one-time acceptable preference relation with better consistency, to achieve a more acceptable effect for decision-makers. Additionally, the ranking results obtained using the two methods differ significantly. For these reasons, we perform the following analysis. (1) The ranking results of Operations 1 and 3 are identical; thus, the proposed ranking method is reasonable and effective. (2) The consistency indices of Operations 1 and 2 differ significantly. Thus, we infer that the difference between the ranking results of Operations 1 and 2 is due to the difference in consistency between $\tilde{R}_{1}$ and $\tilde{R}_{2}$. The consistency level of the matrix $\tilde{R}_{1}$ with acceptable consistency obtained via Zhang et al.'s method [47] is far from consistency threshold under the proposed consistency checking standard, which is unreasonable and leads to an incorrect ranking result. We can verify this through the following logical judgment.

(1) For the matrix $\tilde{R}_{1}$ obtained via Zhang et al.'s method [47], we make a logical judgment based on the evaluation value and the transitivity of the preference relation. If necessary, we use the expected value as an assistant. According to the first row of $\tilde{R}_{1}$, we obtain $x_{1} \succ x_{2} \succ x_{3} \succ x_{4} \sim x_{5}$; according to the second row of $\tilde{R}_{1}$, we obtain $x_{5} \succ x_{1} \succ x_{2} \succ x_{4} \succ x_{3}$; according to the third row of $\tilde{R}_{1}$, we obtain $x_{4} \succ x_{2} \succ x_{1} \succ x_{5} \succ x_{3}$; according to the fourth row of $\tilde{R}_{1}$, we obtain $x_{1} \succ x_{2} \succ x_{4} \succ x_{3} \succ x_{5}$; according to the fifth row of $\tilde{R}_{1}$, we obtain $x_{4} \succ x_{1} \succ x_{5} \succ x_{3} \succ x_{2}$. These five rankings are completely different; thus, there are many logical contradictions in $\tilde{R}_{1}$, which is far from reaching an acceptable consistent preference relation. (2) For the matrix $\tilde{R}_{2}$ obtained via the proposed algorithm, we make logical judgments in the same manner. We obtain the following five ranking results: $x_{1} \succ x_{2} \succ x_{4} \succ x_{3} \succ x_{5}$, $x_{1} \succ x_{2} \succ x_{4} \succ x_{3} \succ x_{5}, \quad x_{1} \succ x_{2} \succ x_{4} \succ x_{3} \succ x_{5}, x_{1} \succ x_{2} \succ x_{4} \succ x_{3} \succ x_{5}$, and $x_{1} \succ x_{4} \succ x_{2} \succ x_{3} \succ x_{5}$, respectively. The five results are relatively consistent; thus, $\tilde{R}_{2}$ is more logical and closer to the completely consistent preference relation. Therefore, the ranking result obtained via the proposed algorithm is more reasonable and accurate.

\section{(2) Comparisons with Gao et al.'s method [8] and Song and Hu's method [27]}

We compared the proposed algorithm with the methods of $[8,27]$ considering the expected additive consistency and expected multiplicative consistency of InPLPRs. The comparison of this subsection is mainly divided into two parts. First, we compare the information completion mechanisms of these methods. Because Gao et al.'s method [8] needs the specific background of the energy emergency, we compare the proposed algorithm with Song and Hu's method [27] using the following operations. Operation 1: We employ the Song and Hu's method [27] to deal with the PLPR $H$ whose data are from the matrix $H^{2}$ in [27] and obtain CPLPR $\bar{H}_{1}$. Operation 2: We employ the proposed algorithm to deal with $H$ and obtain CPLPR $\bar{H}_{2}$. Then, we use the consistency formulas from [27] and the present study to calculate the consistency indices of $\bar{H}_{1}$ and $\bar{H}_{2}$, respectively. The comparison results are presented in Table 2.

Then, to compare the consistency improvement mechanisms of the methods, we performed the following experiment. Using Song and Hu's method [27], Gao et al.'s method [8], and the proposed algorithm (let the consistency threshold be 0.1 and 0.06 , respectively) to simultaneously deal with the inconsistent CPLPR matrix $\bar{H}_{1}$, four matrices are obtained with improved consistency $\left(\tilde{H}_{1}, \tilde{H}_{2}, \tilde{H}_{3}\right.$, and $\tilde{H}_{3}^{\prime}$ ), along with their ranking. We use the consistency measures from [27], [8], and the present study 
to calculate the consistency indices of $\tilde{H}_{1}, \tilde{H}_{2}, \tilde{H}_{3}$, and $\tilde{H}_{3}^{\prime}$. Additionally, we calculate the mean deviation $\theta$ of these adjusted results and count the adjusted LTs and rounds. The comparison results are presented in Table 3 . The matrices $H, \bar{H}_{1}, \bar{H}_{2}, \tilde{H}_{1}, \tilde{H}_{2}, \tilde{H}_{3}$, and $\tilde{H}_{3}^{\prime}$ are presented in Appendix II. The incomplete probabilistic linguistic information processed by Song and Hu's method [27] essentially belongs to the category of the MLT-InPLTS proposed herein.

Table 2. CIs of $\bar{H}_{1}$ and $\bar{H}_{2}$ obtained using different consistency measures

\begin{tabular}{ccc}
\hline Matrices & $\overline{C I}_{A}(0.98 \uparrow)$ & $\overline{C I}_{C}(0.1 \downarrow)$ \\
\hline $\bar{H}_{1}$ & 0.979 & 0.363 \\
$\bar{H}_{2}$ & 0.994 & 0.192 \\
\hline
\end{tabular}

Table 3. Comparison of the three methods

\begin{tabular}{|c|c|c|c|c|c|c|c|}
\hline \multirow{2}{*}{$\begin{array}{l}\text { Matrices obtained } \\
\text { via three methods }\end{array}$} & \multirow{2}{*}{ Ranking } & \multicolumn{3}{|c|}{ CIs obtained via different consistency formulas } & \multirow{2}{*}{$\theta$} & \multirow{2}{*}{$\begin{array}{l}\text { Adjusted } \\
\text { LTs }\end{array}$} & \multirow{2}{*}{$\begin{array}{l}\text { Adjusted } \\
\text { round }\end{array}$} \\
\hline & & $\overline{C I}_{A}^{i}(0.98 \uparrow)$ & $\overline{C I}_{B}^{i}(0.01 \downarrow)$ & $\overline{C I}_{C}^{i}(0.1 \downarrow)$ & & & \\
\hline$\tilde{H}_{1}$ & $A_{4} \succ A_{3} \succ A_{1} \succ A_{2}$ & $0.985(\mathrm{Y})$ & $0.142(\mathrm{~N})$ & $0.211(\mathrm{~N})$ & 0.011 & 2 & 2 \\
\hline$\tilde{H}_{2}$ & $A_{3} \succ A_{4} \succ A_{1} \succ A_{2}$ & $0.999(\mathrm{Y})$ & $0.000(\mathrm{Y})$ & $0.012(\mathrm{Y})$ & 0.029 & 9 & 4 \\
\hline$\tilde{H}_{3}$ & $A_{4} \succ A_{3} \succ A_{1} \succ A_{2}$ & $0.995(\mathrm{Y})$ & $0.072(\mathrm{~N})$ & $0.100(\mathrm{Y})$ & 0.010 & 0 & 1 \\
\hline$\tilde{H}_{3}^{\prime}$ & $A_{3} \succ A_{4} \succ A_{1} \succ A_{2}$ & $0.996(Y)$ & $0.045(\mathrm{~N})$ & $0.060(\mathrm{Y})$ & 0.012 & 0 & 1 \\
\hline
\end{tabular}

Note: In Tables 2 and 3, $\overline{C I}_{A}^{i}(i=1,2,3)$ represents the consistency index obtained via Song and Hu's method [27], $\overline{C I}_{B}^{i}(i=1,2,3)$ represents the consistency index obtained via Gao et al.'s method [8], and $\overline{C I}_{C}^{i}(i=1,2,3)$ represents the consistency index obtained via the proposed method. The 0.98 and 0.01 in parentheses are the consistency thresholds for the corresponding methods. $\uparrow$ indicates that a larger $C I$ corresponds to a more consistent preference matrix, and $\downarrow$ indicates that a smaller $C I$ corresponds to a more consistent preference matrix. $\mathrm{Y}$ indicates that the matrix has acceptable consistency under its standard, and $\mathrm{N}$ indicates the opposite.

As indicated by Table 2, the mechanism for estimating missing information in the proposed algorithm is more accurate than that of Song and Hu's method [27], because the consistency of $\bar{H}_{2}$ obtained by the proposed method is better under any consistency checking standard. From Table 2, comparing the results for $\tilde{H}_{1}$ obtained via Song and Hu's method [27] and the results for $\tilde{H}_{3}$ obtained via the proposed method reveals that although the two rankings are identical, the proposed method can achieve better consistency with less modification rounds and adjusted LTs and can reduce the information distortion caused by the adjustment process. Additionally, according to the consistency criterion of this paper, $\tilde{H}_{2}$ is not an acceptable consistent preference matrix. Comparing the results for $\tilde{H}_{2}$ obtained via Gao et al.'s method [8] and the results for $\tilde{H}_{3}$ reveals that the two rankings are slightly different. This is because $\tilde{H}_{2}$ has better consistency, but the cost of obtaining it is that a large number of LTs are modified in the process of multi-round adjustment, which causes considerable information distortion. Therefore, we reduce the consistency threshold to obtain the matrix $\tilde{H}_{3}^{\prime}$, and compared with the results for $\tilde{H}_{2}$ and $\tilde{H}_{3}^{\prime}$, we find that although the consistency level of $\tilde{H}_{3}^{\prime}$ does not satisfy the threshold in [8], we obtain the same ranking result. This suggests that the $\tilde{H}_{1}$ obtained via Song and Hu's method [27] and its ranking results are not sufficiently accurate. Furthermore, the adjustment cost (the number of adjusted rounds and the number of adjusted LTs) and information distortion are reduced using the proposed consistency improvement algorithm.

\subsubsection{Comparisons with methods considering possibility distribution generation for HFLPRs}

Because the proposed algorithm can also solve decision-making problems in a hesitant fuzzy linguistic preference environment, we compare it with $\mathrm{Wu}$ and Xu's method considering the possibility distribution generation for HFLPRs. The HFLTSs considering the possibility distribution is a special case of InPLTSs, 
i.e., MP-InPLTSs, in which the probabilities of all the LTs are unknown. In view of the research on the generation methods of the possibility distribution for HFLTSs [3, 11, 38], a comparison of the two modules is performed in this section.

First, we compare the proposed probability completion method with the generation methods of the possibility distribution for HFLTSs. The main generation methods include M1, i.e., Wu and Xu's method [38]; M2, i.e., Chen et al.'s method [3] using the normal probability density function (PDF); M3, i.e., Chen et al.'s method [3] using the exponential PDF; M4, i.e., Hao and Chiclana's method [11] considering the linguistic quantifier modeling the soft majority concept "most"; M5, i.e., Hao and Chiclana's method [11] using the regular increasing monotone quantifier with an exponential generating function. For the convenience of the comparison, we denote the proposed method as M6. Because only [38] and this paper involve the study of the preference relationship, we take Example 1 in [38] as the experimental case. The LTSs used in [38] are the subscript-symmetric linguistic evaluation scale $\left\{s_{-\tau}, \ldots, s_{-1}, s_{0}, s_{1}, \ldots, s_{\tau}\right\}$. Therefore, before dealing with Example 1, data conversion is performed for the method of [11] and the proposed method; however, these operations do not affect the experimental results. Additionally, for the parameters related to the attitude in $[3,11]$, the neutral attitude is adopted. The six aforementioned methods are used to generate the probability distribution of the experimental case and obtain six preference relationship matrices, which are denoted as $\bar{B}_{1}, \bar{B}_{2}, \bar{B}_{3}, \bar{B}_{4}, \bar{B}_{5}$, and $\bar{B}_{6}$ (Appendix III). Then, the consistency measures of [38] and this paper (denoted as $C 1$ and $C 2$, respectively) are used to check the consistency levels of these matrices. The experimental results are shown in Fig. 3.

As indicated by Fig. 3, the generating probability distribution for HFLPRs of the proposed method has the best consistency; that is, its logical relationship is the most reasonable. The experimental results suggest that although these existing probability distribution generation methods may have good results in solving some MADM problems, they are inadequate for solving PDM problems. Additionally, we find that for the same matrix, the consistency index obtained via the proposed method is significantly larger than that obtained via the method of [38] based on the additive consistency (by a factor of almost 10). This indicates that the consistency checking standard of this study is more stringent than that of [38]. We performed an experiment to answer the following questions: Which of the two standards is more accurate? Which is the better consistency improvement mechanism? With these questions in mind, we perform the following experiment.

The proposed algorithm is used to deal with Example 1 in [38]. For the convenience of the comparison, we set $\overline{C I}=0.5$ and $\overline{C I}=1$ when we use the proposed algorithm to adjust the consistency. The acceptable consistent matrices obtained are denoted as $\tilde{B}_{2}$ and $\tilde{B}_{3}$. The acceptable consistent matrix obtained via Wu and Xu's method [38] is denoted as $\tilde{B}_{1}$. The two consistency indices of [38] and this paper are employed to check the consistency level of $\tilde{B}_{1}, \tilde{B}_{2}$, and $\tilde{B}_{3}$, and the proposed ranking method is used to rank all the alternatives (because ranking methods were not studied in [38]). Additionally, the mean deviations $\left(\theta_{i}\right)$ of these adjusted matrices are calculated, and the adjusted LTs and rounds are counted. The matrices $\tilde{B}_{1}, \tilde{B}_{2}$, and $\tilde{B}_{3}$ are presented in Appendix III. The comparison results are presented in Table 4.

Comparing the results obtained via $\mathrm{Wu}$ and Xu's method [38] and the proposed method $(\overline{C I}=1)$ reveals that the proposed algorithm can achieve better consistency with less adjustment rounds, adjusted LTs, and information distortion. The difference in the ranking results obtained via the two methods is 
explained as follows: the consistency of the matrix $\tilde{B}_{1}$ obtained via Wu and Xu's method [38] does not reach the ideal level; that is, there is a lack of sufficient logical relations. This conjecture can be verified by comparing the ranking results of $\tilde{B}_{1}$ and $\tilde{B}_{2}$; i.e., the matrix $\tilde{B}_{2}$ has the same ranking as $\tilde{B}_{3}$, and $\tilde{B}_{2}$ has better consistency according to any consistency measure. Therefore, the proposed consistency improvement algorithm can obtain more reasonable ranking results.

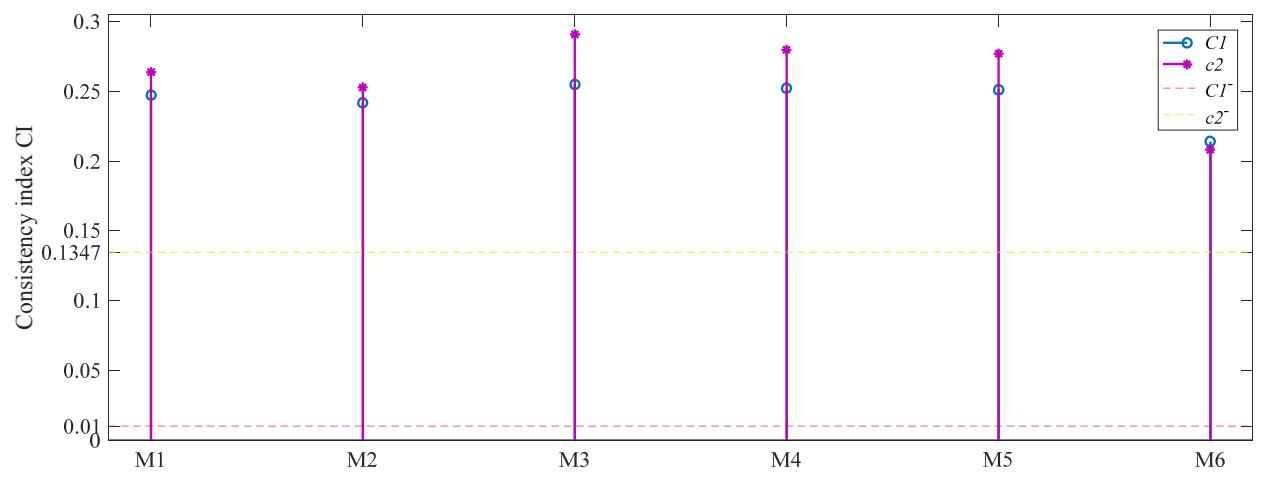

Fig. 3. Consistency for different matrices obtained via different methods under the two test standards

Note: for better visualization, we reduced the value of $C 2$ by 10 times and expressed it as $c 2 . \mathrm{Cl}^{-}$and $c 2^{-}$represent the threshold.

Table 4. Experimental results for the different methods

\begin{tabular}{ccccccccc}
\hline Methods & \multicolumn{2}{c}{ Consistency index of [41] } & Current consistency index & Ranking & $\begin{array}{c}\text { Adjusted } \\
\text { LTs }\end{array}$ & $\begin{array}{c}\text { Adjusted } \\
\text { round }\end{array}$ & $\theta$ \\
\hline $\begin{array}{c}\text { Wu and Xu's method } \\
\text { [38] }\end{array}$ & 0.247 & 0.118 & 2.637 & 1.0 & $x_{1} \succ x_{4} \succ x_{3} \succ x_{2}$ & 5 & 3 & 1.125 \\
$\begin{array}{c}\text { Proposed method } \\
(\overline{C I}=0.5)\end{array}$ & 0.214 & 0.067 & 2.081 & 0.5 & $x_{1} \succ x_{2} \succ x_{4} \succ x_{3}$ & 3 & 1 & 0.080 \\
$\begin{array}{c}\text { Proposed method } \\
(\overline{C I}=1)\end{array}$ & 0.214 & 0.116 & 2.081 & 1.0 & $x_{1} \succ x_{2} \succ x_{4} \succ x_{3}$ & 2 & 1 & 0.050 \\
\hline
\end{tabular}

Note: For the convenience of the comparison, we set $\overline{C I}=0.5$ and 1 when using the proposed method to improve the consistency.

\subsubsection{Discussion}

The detailed comparative analysis revealed that the proposed algorithm has the following advantages over existing methods.

(1) The information estimation mechanism for InPLPRs is more scientific and accurate. Comparing the results of CPLPRs obtained via Zhang et al.'s method [47] considering traditional standardization and Song and Hu's method [27] considering optimization based on the consistency measure revealed that the CPLPRs obtained via the proposed method had better consistency performance. This is because the information completion mechanisms in [47] and [27] can only deal with one type of incomplete information, i.e., where the sum of the probabilities of all the possible LTs is $<1$. These methods assign the missing probability to the existing LTs according to the proportion of the existing probability of LTs and the consistency criteria, which are all unreasonable and arbitrary. In actual decision-making, the incomplete probability arises from the lack of opinions or the hesitation of decision-makers. Therefore, we believe that the partial missing probability should belong to the scope of the original domain, rather than exist only in the given LTs. Additionally, there are two ways to deal with probabilistic linguistic preference information which has multiple or even all LTs whose probabilities are not given. One is the method given in $[8,9]$ based on the EFTA technology which has obvious application limitations. The other is to consider the possibility distribution generation in $[3,11,38]$, but this kind of method has obvious inaccuracy when dealing with PDM problems. The information completion mechanism in this paper divides the InPLTSs into two categories, and then uses a two-stage optimization model to estimate 
missing information based on the principle of consistency and uncertainty. This method can obtain the complete probabilistic linguistic information with the minimum uncertainty under the premise of ensuring the best consistency of CPLPR. In addition, this method can also deal with the PDM problems based on hesitant fuzzy linguistic information. Therefore, the information completion mechanism in the proposed algorithm is more reasonable and universal.

(2) The consistency checking and improvement mechanism is more efficient. Comparing the ordinal consistency based on preference relation graph in [47] and the additive consistency in [38], the consistency index based on the multiplicative consistency in this paper has more accurate measurement effect, and can help to obtain an acceptable preference relationship which has higher logical rationality. For the research of consistency correction strategy, the mainstream methods are to use the reduplicative iteration for local adjustment, such as the methods in [9, 27, 38, 47]. However, these methods cannot give an accurate adjustment range, which may make the adjusted matrix deviate far from the original matrix, that is, there is a lot of information distortion in these methods. In addition, the iterative method is time-consuming, and is not suitable for solving some emergency decision-making problems. Recently, the optimization model based on the minimum information distortion have been mentioned and studied for PLPRs in [15]. However, it is global and large-scale modification, which makes the adjusted results difficult to satisfy decision-makers. What is different is that this paper proposes a multi-stage optimization model based on the principle of the minimum information distortion, the least number of adjusted LTs and the minimum information uncertainty to modify the consistency of unacceptable CPLPR. By using the proposed consistency improvement algorithm, we can obtain a CPLPR with acceptable consistency by adjusting as few LTs as possible under the premise of minimizing information distortion in a short period of time, and ensure that the obtained CPLPR have less uncertainty of information. The above comparative analysis in Section 5.3 also verified these advantages of the proposed method.

(3) The ranking method proposed in this paper is simple and effective. The ranking methods in $[15,16$, 21, 46-48] use aggregation operators of PLTSs to rank alternatives, which are not only complex but also easy to cause information distortion owing to the immature operation rules of PLTSs. For example, in some methods, each PLTS to be operated should have the same number of elements, which is difficult to be obtained in reality. If the elements in initial PLTSs are deleted or filled by artificial method, this operation is easy to cause information distortion. Research shows that it is an effective method to rank alternatives based on priority weights. However, the usual methods in $[8,9,19,27,41]$ are based on the optimization models solved by software to obtain the priority weights. The difference is that this paper uses the mathematical method to solve the programming model, and then derives the priority weights through a general mathematical formula to rank the alternatives. Thus, the proposed ranking method is easier to operate.

\section{Conclusions}

InPLPSs can effectively describe the incomplete information caused by the lack of opinion or the hesitation of experts, so they are more suitable for solving PDM problems. This paper focuses on how to construct a decision-making algorithm based on the consistency of InPLPRs. It mainly consists of three parts: estimating missing information, checking and improving consistency, and ranking. First of all, a two-stage optimization model was built to estimate missing information based on the expected multiplicative consistency of InPLPR. Then, the consistency index of InPLPR was defined to check consistency level. In order to obtain a CPLPR with acceptable consistency, a multi-stage 
consistency-improving optimization model was constructed. Finally, in order to rank the alternatives, a solved. The proposed algorithm in this paper has the following advantages than some existing methods:

(1) The information completion mechanism is more reasonable and effective, which considers the principles of the best consistency and the minimum uncertainty of information. (2) The consistency measure is more accurate, and the consistency improvement mechanism is more effective. The consistency improving algorithm can obtain the acceptable consistent CPLPR based on the minimum information distortion, the least number of adjusted LTs and the minimum information uncertainty. Additionally, this algorithm is time-saving. (3) The proposed method of deriving priority weights for ranking alternatives is efficient and easier to operate.

It should be noted that this paper only considered the expected multiplicative consistency of InPLPR. Therefore, when there are too many alternatives or incomplete information elements, the complexity of the models will become very high, which brings great challenges to obtaining optimal solution. So how to simplify the models will be the focus of future research, such as using additive consistency. In addition, how to solve the consensus problems with InPLPRs for GDM is also deserves to be studied.

\section{References}

[1] J. Borwein, A.S. Lewis, Convex Analysis and Nonlinear Optimization: Theory and Examples, Springer Science \& Business Media, (2010).

[2] Z.S. Chen, K.S. Chin, Y.L. Li, Y. Yang, Proportional hesitant fuzzy linguistic term set for multiple criteria group decision making, Information Sciences, 357(2016):61-87.

[3] Z.S. Chen, K.S. Chin, N.Y. Mu, S.H. Xiong, J.P. Chang, Y. Yang, Generating HFLTS possibility distribution with an embedded assessing attitude, Information Sciences, 394(2017):141-166.

[4] F. Chiclana, E. Herrera-Viedma, S. Alonso, F. Herrera, Cardinal consistency of reciprocal preference relations: a characterization of multiplicative transitivity, Ieee Transactions on Fuzzy Systems, 17(1) (2008):14-23.

[5] T.M. Cover, J.A. Thomas, Elements of Information Theory (Second Edition), New Jersey: John Wiley \& Sons, (2006).

[6] Y.C. Dong, E. Herrera-Viedma, Consistency-driven automatic methodology to set interval numerical scales of 2-tuple linguistic term sets and its use in the linguistic GDM with preference relation, IEEE transactions on cybernetics, 45(4) (2014):780-792.

[7] Y.C. Dong, Y.F. Xu, H.Y. Li, On consistency measures of linguistic preference relations, European Journal of Operational Research, 189(2) (2008):430-444.

[8] J. Gao, Z.S. Xu, Z.L. Liang, H.C. Liao, Expected consistency-based emergency decision making with incomplete probabilistic linguistic preference relations, Knowledge-Based Systems, 176(2019):15-28.

[9] J. Gao, Z.S. Xu, P.J. Ren, H.C. Liao, An emergency decision making method based on the multiplicative consistency of probabilistic linguistic preference relations, International Journal of Machine Learning Cybernetics, 18(7) (2018):1613-1629.

[10] S. Greco, B. Matarazzo, R. Slowinski, Rough sets theory for multicriteria decision analysis, European Journal of Operational Research, 129(1) (2001):1-47.

[11] J.J. Hao, F. Chiclana, Attitude Quantifier Based Possibility Distribution Generation Method for Hesitant Fuzzy Linguistic Group Decision Making, Information Sciences, 518(2020):341-360.

[12] F. Herrera, E. Herrera-Viedma, Choice functions and mechanisms for linguistic preference relations, European Journal of Operational Research, 120(1) (2000):144-161.

[13] B. Li, Y.X. Zhang, Z.S. Xu, The Aviation Technology Two-Sided Matching with the Expected Time 
Based on the Probabilistic Linguistic Preference Relations, Journal of the Operations Research Society of China, 8(1) (2020):45-77.

[14] C.C. Li, R.M. Rodriguez, L. Martinez, Y.C. Dong, F. Herrera, Consistency of hesitant fuzzy linguistic preference relations: An interval consistency index, Information Sciences, 432(2018):347-361.

[15] P. Liang, J. Hu, B. Li, Y. Liu, X. Chen, A group decision making with probability linguistic preference relations based on nonlinear optimization model and fuzzy cooperative games, Fuzzy Optimization and Decision Making, (2020):1-30. https://doi.org/10.1007/s10700-020-09329-6

[16] H.C. Liao, X.Y. Peng, X.J. Gou, Medical Supplier Selection with a Group Decision-Making Method Based on Incomplete Probabilistic Linguistic Preference Relations, International Journal of Fuzzy Systems, (2020):1-15. https://doi.org/10.1007/s40815-020-00885-y

[17] H.C. Liao, Z.S. Xu, M.M. Xia, MULTIPLICATIVE CONSISTENCY OF HESITANT FUZZY PREFERENCE RELATION AND ITS APPLICATION IN GROUP DECISION MAKING, International Journal of Information Technology and Decision Making, 13(01) (2014):47-76.

[18] P.D. Liu, F. Teng, Probabilistic linguistic TODIM method for selecting products through online product reviews, Information Sciences, 485(2019):441-455.

[19] S.Z. Luo, H.Y. Zhang, J.Q. Wang, L. Li, Group decision-making approach for evaluating the sustainability of constructed wetlands with probabilistic linguistic preference relations, Journal of the Operational Research Society, 70(12) (2019):2039-2055.

[20] X. Mi, H. Liao, X. Wu, Z. Xu, Probabilistic linguistic information fusion: A survey on aggregation operators in terms of principles, definitions, classifications, applications, and challenges, International Journal of Intelligent Systems, 35(3) (2020):529-556.

[21] R.X. Nie, J.Q. Wang, Prospect Theory-Based Consistency Recovery Strategies with Multiplicative Probabilistic Linguistic Preference Relations in Managing Group Decision Making, Arabian Journal for Science and Engineering, 45(3) (2020):2113-2130.

[22] S. Orlovsky, Decision-making with a fuzzy preference relation, Fuzzy Sets and Systems, 1(3) (1978):155-167.

[23] Q. Pang, H. Wang, Z.S. Xu, Probabilistic linguistic linguistic term sets in multi-attribute group decision making, Information Sciences, 369(2016):128-143.

[24] R.M. Rodriguez, L. Martinez, F. Herrera, Hesitant Fuzzy Linguistic Term Sets for Decision Making, Ieee Transactions on Fuzzy Systems, 20(1) (2012):109-119.

[25] T.L. Saaty, A scaling method for priorities in hierarchical structures, Journal of mathematical psychology, 15(3) (1977):234-281.

[26] Y.M. Song, Deriving the priority weights from probabilistic linguistic preference relation with unknown probabilities, PLoS One, 13(12) (2018):e0208855.

[27] Y.M. Song, J. Hu, Large-scale group decision making with multiple stakeholders based on probabilistic linguistic preference relation, Applied Soft Computing, 80(2019):712-722.

[28] T. Tanino, Fuzzy preference orderings in group decision making, Fuzzy Sets and Systems, 12(2) (1984):117-131.

[29] J.F. Tian, Z.M. Zhang, M.H. Ha, An additive consistency and consensus-based approach for uncertain group decision making with linguistic preference relations, Ieee Transactions on Fuzzy Systems, 27(5) (2019):873-887.

[30] S.P. Wan, L.G. Zhong, J.Y. Dong, A new method for group decision making with hesitant fuzzy preference relations based on multiplicative consistency, Ieee Transactions on Fuzzy Systems, 28(7) (2020):1449-1463.

[31] H. Wang, Extended hesitant fuzzy linguistic term sets and their aggregation in group decision 
making, International Journal of Computational Intelligence Systems, 8(1) (2015):14-33.

[32] H. Wang, Z.S. Xu, Some consistency measures of extended hesitant fuzzy linguistic preference relations, Information Sciences, 297(2015):316-331.

[33] J.H. Wang, J.Y. Hao, A new version of 2-tuple. fuzzy linguistic, representation model for computing with words, Ieee Transactions on Fuzzy Systems, 14(3) (2006):435-445.

[34] X.T. Wang, E. Triantaphyllou, Ranking irregularities when evaluating alternatives by using some ELECTRE methods, Omega-International Journal of Management Science, 36(1) (2008):45-63.

[35] Y.M. Wang, Y. Luo, On rank reversal in decision analysis, Mathematical and computer modelling, 49(5-6) (2009):1221-1229.

[36] J. Wu, F. Chiclana, Non-dominance and attitudinal prioritisation methods for intuitionistic and interval-valued intuitionistic fuzzy preference relations, Expert Systems with Applications, 39(18) (2012):13409-13416.

[37] X.L. Wu, H.C. Liao, An approach to quality function deployment based on probabilistic linguistic term sets and ORESTE method for multi-expert multi-criteria decision making, Information Fusion, 43(2018):13-26.

[38] Z.B. Wu, J.P. Xu, Managing consistency and consensus in group decision making with hesitant fuzzy linguistic preference relations, Omega-International Journal of Management Science, 65(2016):28-40.

[39] Z.B. Wu, J.P. Xu, Possibility Distribution-Based Approach for MAGDM With Hesitant Fuzzy Linguistic Information, IEEE transactions on cybernetics, 46(3) (2016):694-705.

[40] M.M. Xia, Z.S. Xu, Z. Wang, Multiplicative consistency-based decision support system for incomplete linguistic preference relations, International Journal of Systems Science, 45(3) (2014):625-636.

[41] W.Y. Xie, Z.S. Xu, Z.L. Ren, An Analysis on the Influence of Chinese "New Four Inventions" Under the Incomplete Hybrid Probabilistic Linguistic Environment, International Journal of Fuzzy Systems, 21(5) (2019):1349-1366.

[42] Y.J. Xu, L. Chen, R.M. Rodriguez, F. Herrera, H.M. Wang, Deriving the priority weights from incomplete hesitant fuzzy preference relations in group decision making, Knowledge-Based Systems, 99(2016):71-78.

[43] Z.S. Xu, A method based on linguistic aggregation operators for group decision making with linguistic preference relations, Information Sciences, 166(1-4) (2004):19-30.

[44] L.A. Zadeh, The concept of a linguistic variable and its application to approximate reasoning-I, Information Sciences, 8(3) (1975):199-249.

[45] G.Q. Zhang, Y.C. Dong, Y.F. Xu, Consistency and consensus measures for linguistic preference relations based on distribution assessments, Information Fusion, 17(2014):46-55.

[46] Y.X. Zhang, Z.S. Xu, H.C. Liao, A consensus process for group decision making with probabilistic linguistic preference relations, Information Sciences, 414(2017):260-275.

[47] Y.X. Zhang, Z.S. Xu, H.C. Liao, An ordinal consistency-based group decision making process with probabilistic linguistic preference relation, Information Sciences, 467(2018):179-198.

[48] Y.X. Zhang, Z.S. Xu, H. Wang, H.C. Liao, Consistency-based risk assessment with probabilistic linguistic preference relation, Applied Soft Computing, 49(2016):817-833.

[49] Z.M. Zhang, C. Wu, On the use of multiplicative consistency in hesitant fuzzy linguistic preference relations, Knowledge Based Systems, 72(1) (2014):13-27.

[50] W. Zhou, Z.S. Xu, Probability Calculation and Element Optimization of Probabilistic Hesitant Fuzzy Preference Relations Based on Expected Consistency, Ieee Transactions on Fuzzy Systems, 26(3) (2018):1367-1378. 


\section{Appendix}

Appendix I.

Note: to fit the page width, in the following matrices, we only give the elements in the upper triangular matrix and the corresponding elements of lower triangular matrix are omitted, denoted by the symbol “",- ,

$$
\begin{aligned}
& R^{\text {original }}=\left[\begin{array}{lllll}
\left\{s_{3}(1)\right\} & \left\{s_{3}(0.1), s_{4}(0.4)\right\} & \left\{s_{2}(0.6), s_{5}(0.2)\right\} & \left\{s_{5}(0.7)\right\} & \left\{s_{4}(0.4), s_{6}(0.4)\right\} \\
- & \left\{s_{3}(1)\right\} & \left\{s_{5}(0.6)\right\} & \left\{s_{4}(0.3), s_{5}(0.4)\right\} & \left\{s_{0}(0.3), s_{3}(0.2)\right\} \\
- & - & \left\{s_{3}(1)\right\} & \left\{s_{0}(0.2), s_{5}(0.5)\right\} & \left\{s_{2}(0.9)\right\} \\
- & - & - & \left\{s_{3}(1)\right\} & \left\{s_{4}(0.2), s_{6}(0.8)\right\} \\
- & - & - & - & \left\{s_{3}(1)\right\}
\end{array}\right] \\
& \bar{R}_{1}=\left[\begin{array}{lllll}
\left\{s_{3}(1)\right\} & \left\{s_{3}(\mathbf{0 . 2}), s_{4}(\mathbf{0 . 8})\right\} & \left\{s_{2}(\mathbf{0 . 7 5}), s_{5}(\mathbf{0 . 2 5})\right\} & \left\{s_{5}(\mathbf{1})\right\} & \left\{s_{4}(\mathbf{0 . 5}), s_{6}(\mathbf{0 . 5})\right\} \\
- & \left\{s_{3}(1)\right\} & \left\{s_{5}(\mathbf{1})\right\} & \left\{s_{4}(\mathbf{0 . 4 3}), s_{5}(\mathbf{0 . 5 7})\right\} & \left\{s_{0}(\mathbf{0 . 6}), s_{3}(\mathbf{0 . 4})\right\} \\
- & - & \left\{s_{3}(1)\right\} & \left\{s_{0}(\mathbf{0 . 2 9}), s_{5}(\mathbf{0 . 7 1})\right\} & \left\{s_{2}(\mathbf{1})\right\} \\
- & - & - & \left\{s_{3}(1)\right\} & \left\{s_{4}(0.2), s_{6}(0.8)\right\} \\
- & - & - & - & \left\{s_{3}(1)\right\}
\end{array}\right]
\end{aligned}
$$$$
\tilde{R}_{1}=\left[\begin{array}{lllll}
\left\{s_{3}(1)\right\} & \left\{s_{3}(0.2), s_{4}(0.8)\right\} & \left\{s_{5}(0.25), s_{4}(0.75)\right\} & \left\{s_{5}(1)\right\} & \left\{s_{4}(0.5), s_{6}(0.5)\right\} \\
- & \left\{s_{3}(1)\right\} & \left\{s_{5}(1)\right\} & \left\{s_{4}(0.43), s_{5}(0.57)\right\} & \left\{s_{0}(0.6), s_{3}(0.4)\right\} \\
- & - & \left\{s_{3}(1)\right\} & \left\{s_{0}(0.29), s_{1}(0.71)\right\} & \left\{s_{2}(1)\right\} \\
- & - & - & \left\{s_{3}(1)\right\} & \left\{s_{4}(0.2), s_{6}(0.8)\right\} \\
- & - & - & - & \left\{s_{3}(1)\right\}
\end{array}\right]
$$$$
\bar{R}_{2}=\left[\begin{array}{lllll}
\left\{s_{3}(1)\right\} & \left\{\boldsymbol{s}_{2.806}(\mathbf{0 . 5}), s_{3}(0.1), s_{4}(0.4)\right\} & \left\{s_{2}(0.6), s_{5}(0.2), \boldsymbol{s}_{\mathbf{6}}(\mathbf{0 . 2})\right\} & \left\{\boldsymbol{s}_{\mathbf{0}}(\mathbf{0 . 3}), s_{5}(0.7)\right\} & \left\{\boldsymbol{s}_{\mathbf{0}}(\mathbf{0 . 2}), s_{4}(0.4), s_{6}(0.4)\right\} \\
- & \left\{s_{3}(1)\right\} & \left\{\boldsymbol{s}_{\mathbf{1 . 4 5 5}}(\mathbf{0 . 4}), s_{5}(0.6)\right\} & \left\{\mathbf{s}_{\mathbf{0}}(\mathbf{0 . 3}), s_{4}(0.3), s_{5}(0.4)\right\} & \left\{s_{0}(0.3), s_{3}(0.2), \mathbf{s}_{\mathbf{6}}(\mathbf{0 . 5})\right\} \\
- & - & \left\{s_{3}(1)\right\} & \left\{s_{0}(0.2), s_{0.3902}(\mathbf{0 . 3}), s_{5}(0.5)\right\} & \left\{s_{2}(0.9), \boldsymbol{s}_{\mathbf{6}}(\mathbf{0 . 1})\right\} \\
- & - & - & \left\{s_{3}(1)\right\} & \left\{s_{4}(0.2), s_{6}(0.8)\right\} \\
- & - & - & - & \left\{s_{3}(1)\right\}
\end{array}\right]
$$$$
\tilde{R}_{2}=\left[\begin{array}{lllll}
\left\{s_{3}(1)\right\}\left\{s_{2.807}(\mathbf{0 . 0 1}), s_{3}(\mathbf{0 . 6 8 5}), s_{4}(\mathbf{( 0 . 3 0 5 3})\right\} & \left\{s_{2}(\mathbf{0 . 3 8 2}), s_{5}(\mathbf{( 0 . 6 0 8}), s_{6}(\mathbf{0 . 0 1})\right\} & \left\{s_{0}(0.3), s_{5}(0.7)\right\} & \left\{s_{0}(\mathbf{0 . 0 1}), s_{4}(\mathbf{0 . 9 7}), s_{6}(\mathbf{0 . 0 2})\right\} \\
- & \left\{s_{3}(1)\right\} & \left\{s_{1.45}(0.4), s_{5}(0.6)\right\} & \left\{s_{0}(\mathbf{0 . 2 0 2}), s_{4}(\mathbf{0 . 7 8 8}), s_{5}(\mathbf{0 . 0 1})\right\} & \left\{s_{0}(\mathbf{0 . 0 1}), s_{3}(\mathbf{0 . 7 8}), s_{6}(\mathbf{0 . 2 1})\right\} \\
- & - & \left\{s_{3}(1)\right\} & \left\{s_{0}(\mathbf{0 . 0 1}), s_{0.30}(\mathbf{0 . 5 0 6}), s_{5}(\mathbf{0 . 4 8 4})\right\} & \left\{s_{2}(\mathbf{0 . 7 4 4}), s_{6}(\mathbf{0 . 2 5 6})\right\} \\
- & - & - & \left\{s_{3}(1)\right\} & \left\{\boldsymbol{s}_{3.2 .21}(\mathbf{0 . 9 9}), s_{6}(\mathbf{0 . 0 1})\right\} \\
- & - & - & - & \left\{s_{3}(1)\right\}
\end{array}\right]
$$

Appendix II.

$$
\begin{aligned}
& \bar{H}_{1}=\left[\begin{array}{llll}
\left\{s_{4}(1)\right\}\left\{s_{4}(0.4), s_{5}(0.6)\right\} & \left\{s_{2}(0.5), s_{3}(0.5)\right\} & \left\{s_{3}(\mathbf{0 . 8}), s_{4}(0.2)\right\} \\
- & \left\{s_{4}(1)\right\} & \left\{s_{2}(0.4), s_{3}(\mathbf{0 . 6})\right\}\left\{s_{2}(\mathbf{0 . 5}), s_{3}(0.3), s_{5}(0.2)\right\} \\
- & - & \left\{s_{4}(1)\right\} & \left\{s_{3}(0.4), s_{4}(0.6)\right\} \\
- & - & - & \left\{s_{4}(1)\right\}
\end{array}\right]
\end{aligned}
$$

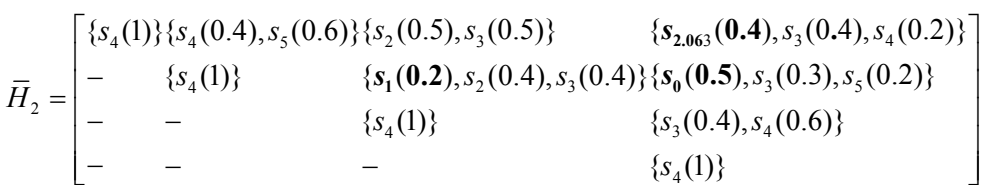

$$
\begin{aligned}
& \tilde{H}_{1}=\left[\begin{array}{llll}
\left\{s_{4}(1)\right\}\left\{s_{4}(0.4), s_{5}(0.6)\right\}\left\{\boldsymbol{s}_{\mathbf{2 . 5 5}}(0.5), \boldsymbol{s}_{\mathbf{3 . 5 5}}(0.5)\right\} & \left\{s_{3}(0.8), s_{4}(0.2)\right\} \\
- & \left\{s_{4}(1)\right\} & \left\{s_{2}(0.4), s_{3}(0.6)\right\} & \left\{s_{2}(0.5), s_{3}(0.3), s_{5}(0.2)\right\} \\
- & - & \left\{s_{4}(1)\right\} & \left\{s_{3}(0.4), s_{4}(0.6)\right\} \\
- & - & - & \left\{s_{4}(1)\right\}
\end{array}\right]
\end{aligned}
$$

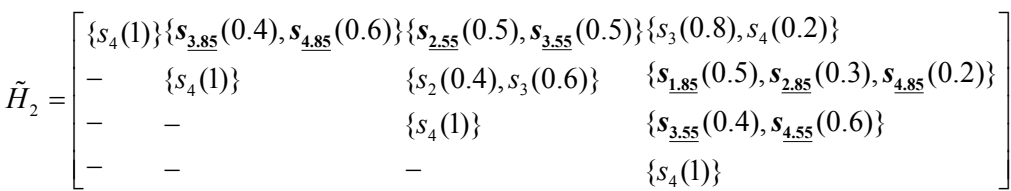


$\tilde{H}_{3}=\left[\begin{array}{llll}\left\{s_{4}(1)\right\}\left\{s_{4}(0.4), s_{5}(0.6)\right\}\left\{s_{2}(\mathbf{0 . 0 1}), s_{3}(\mathbf{0 . 9 9})\right\} & \left\{s_{3}(0.8), s_{4}(0.2)\right\} \\ - & \left\{s_{4}(1)\right\} & \left\{s_{2}(0.4), s_{3}(0.6)\right\} & \left\{s_{2}(\mathbf{0 . 7 8}), s_{3}(\mathbf{0 . 0 1}), s_{5}(\mathbf{0 . 2 1})\right\} \\ - & - & \left\{s_{4}(1)\right\} & \left\{s_{3}(\mathbf{0 . 1 7 8}), s_{4}(\mathbf{0 . 8 2 2})\right\} \\ - & - & - & \left\{s_{4}(1)\right\}\end{array}\right]$

$\tilde{H}_{3}^{\prime}=\left[\begin{array}{llll}\left\{s_{4}(1)\right\}\left\{s_{4}(0.4), s_{5}(0.6)\right\}\left\{s_{2}(\mathbf{0 . 0 1}), s_{3}(\mathbf{0 . 9 9})\right\}\left\{s_{3}(0.8), s_{4}(0.2)\right\} \\ - & \left\{s_{4}(1)\right\} & \left\{s_{2}(0.4), s_{3}(0.6)\right\} & \left\{s_{2}(\mathbf{0 . 3 7 9}), s_{3}(\mathbf{0 . 6 1 1}), s_{5}(\mathbf{0 . 0 1})\right\} \\ - & - & \left\{s_{4}(1)\right\} & \left\{s_{3}(\mathbf{0 . 0 1 9}), s_{4}(\mathbf{0 . 9 8 1})\right\} \\ - & - & - & \left\{s_{4}(1)\right\}\end{array}\right]$

Appendix III.

$$
\begin{aligned}
& \bar{B}_{1}=\left[\begin{array}{llll}
\left\{s_{0}(1)\right\} & \left\{s_{1}(1)\right\} & \left\{s_{2}(0.5), s_{3}(0.5)\right\} & \left\{s_{1}(1)\right\} \\
- & \left\{s_{0}(1)\right\} & \left\{s_{-4}(0.5), s_{-3}(0.5)\right\} & \left\{s_{2}(1)\right\} \\
- & - & \left\{s_{0}(1)\right\} & \left\{s_{-3}(0.5), s_{-2}(0.5)\right\} \\
- & - & - & \left\{s_{0}(1)\right\}
\end{array}\right] \\
& \bar{B}_{2}=\left[\begin{array}{llll}
\left\{s_{0}(1)\right\} & \left\{s_{1}(1)\right\} & \left\{s_{2}(0.595), s_{3}(0.405)\right\} & \left\{s_{1}(1)\right\} \\
- & \left\{s_{0}(1)\right\} & \left\{s_{-4}(0.431), s_{-3}(0.569)\right\} & \left\{s_{2}(1)\right\} \\
- & - & \left\{s_{0}(1)\right\} & \left\{s_{-3}(0.405), s_{-2}(0.595)\right\} \\
- & - & - & \left\{s_{0}(1)\right\}
\end{array}\right] \\
& \bar{B}_{3}=\left[\begin{array}{llll}
\left\{s_{0}(1)\right\} & \left\{s_{1}(1)\right\} & \left\{s_{2}(0.661), s_{3}(0.339)\right\} & \left\{s_{1}(1)\right\} \\
- & \left\{s_{0}(1)\right\} & \left\{s_{-4}(0.661), s_{-3}(0.339)\right\} & \left\{s_{2}(1)\right\} \\
- & - & \left\{s_{0}(1)\right\} & \left\{s_{-3}(0.661), s_{-2}(0.339)\right\} \\
- & - & - & \left\{s_{0}(1)\right\}
\end{array}\right] \\
& \bar{B}_{4}=\left[\begin{array}{llll}
\left\{s_{0}(1)\right\} & \left\{s_{1}(1)\right\} & \left\{s_{2}(0.586), s_{3}(0.414)\right\} & \left\{s_{1}(1)\right\} \\
- & \left\{s_{0}(1)\right\} & \left\{s_{-4}(0.586), s_{-3}(0.414)\right\} & \left\{s_{2}(1)\right\} \\
- & - & \left\{s_{0}(1)\right\} & \left\{s_{-3}(0.586), s_{-2}(0.414)\right\} \\
- & - & - & \left\{s_{0}(1)\right\}
\end{array}\right] \\
& \bar{B}_{5}=\left[\begin{array}{llll}
\left\{s_{0}(1)\right\} & \left\{s_{1}(1)\right\} & \left\{s_{2}(0.6), s_{3}(0.4)\right\} & \left\{s_{1}(1)\right\} \\
- & \left\{s_{0}(1)\right\} & \left\{s_{-4}(0.6), s_{-3}(0.4)\right\} & \left\{s_{2}(1)\right\} \\
- & - & \left\{s_{0}(1)\right\} & \left\{s_{-3}(0.6), s_{-2}(0.4)\right\} \\
- & - & - & \left\{s_{0}(1)\right\}
\end{array}\right] \\
& \bar{B}_{6}=\left[\begin{array}{llll}
\left\{s_{0}(1)\right\} & \left\{s_{1}(1)\right\} & \left\{s_{2}(0.99), s_{3}(0.01)\right\} & \left\{s_{1}(1)\right\} \\
- & \left\{s_{0}(1)\right\} & \left\{s_{-4}(0.01), s_{-3}(0.99)\right\} & \left\{s_{2}(1)\right\} \\
- & - & \left\{s_{0}(1)\right\} & \left\{s_{-3}(0.01), s_{-2}(0.99)\right\} \\
- & - & - & \left\{s_{0}(1)\right\}
\end{array}\right] \\
& \tilde{B}_{1}=\left[\begin{array}{llll}
\left\{s_{0}(1)\right\} & \left\{s_{1}(1)\right\} & \left\{s_{2}(0.5), s_{3}(0.5)\right\} & \left\{s_{1}(1)\right\} \\
- & \left\{s_{0}(1)\right\} & \left\{\boldsymbol{s}_{\underline{-2}}(0.5), \boldsymbol{s}_{-\underline{1}}(0.5)\right\} & \left\{\boldsymbol{s}_{\underline{0}}(0.5), \boldsymbol{s}_{\underline{\mathbf{1}}}(0.5)\right\} \\
- & - & \left\{s_{0}(1)\right\} & \left\{\boldsymbol{s}_{-\underline{-2}}(0.5), \boldsymbol{s}_{-\underline{1}}(0.5)\right\} \\
- & - & - & \left\{s_{0}(1)\right\}
\end{array}\right] \\
& \tilde{B}_{2}=\left[\begin{array}{llll}
\left\{s_{0}(1)\right\} & \left\{s_{1}(1)\right\} & \left\{s_{2}(0.99), s_{3}(0.01)\right\} & \left\{s_{1}(1)\right\} \\
- & \left\{s_{0}(1)\right\} & \left\{s_{-4}(0.01), \underline{s_{1.207}}(0.99)\right\} & \left\{\boldsymbol{s}_{\underline{\mathbf{1 . 8 4 8}}}(1)\right\} \\
- & - & \left\{s_{0}(1)\right\} & \left\{s_{-3}(0.01), \boldsymbol{s}_{\underline{-1.137}}(0.99)\right\} \\
- & - & - & \left\{s_{0}(1)\right\}
\end{array}\right] \\
& \tilde{B}_{3}=\left[\begin{array}{llll}
\left\{s_{0}(1)\right\} & \left\{s_{1}(1)\right\} & \left\{s_{2}(0.99), s_{3}(0.01)\right\} & \left\{s_{1}(1)\right\} \\
- & \left\{s_{0}(1)\right\} & \left\{s_{-4}(0.01), s_{-0.575}(0.99)\right\} & \left\{s_{2}(1)\right\} \\
- & - & \left\{s_{0}(1)\right\} & \left\{s_{-3}(0.01), s_{-\underline{-1.137}}(0.99)\right\} \\
- & - & - & \left\{s_{0}(1)\right\}
\end{array}\right]
\end{aligned}
$$

